\title{
SNP mining in C. clementina BAC end sequences; transferability in the Citrus genus (Rutaceae), phylogenetic inferences and perspectives for genetic mapping
}

Patrick Ollitrault ${ }^{1,2^{*}}$, Javier Terol ${ }^{3}$, Andres Garcia-Lor ${ }^{2}$, Aurélie Bérard ${ }^{4}$, Aurélie Chauveau ${ }^{4}$, Yann Froelicher ${ }^{1}$, Caroline Belzile ${ }^{2}$, Raphaël Morillon ${ }^{1,3}$, Luis Navarro², Dominique Brunel ${ }^{4}$ and Manuel Talon ${ }^{3}$

\begin{abstract}
Background: With the increasing availability of EST databases and whole genome sequences, SNPs have become the most abundant and powerful polymorphic markers. However, SNP chip data generally suffers from ascertainment biases caused by the SNP discovery and selection process in which a small number of individuals are used as discovery panels. The ongoing International Citrus Genome Consortium sequencing project of the highly heterozygous Clementine and sweet orange genomes will soon result in the release of several hundred thousand SNPs. The primary goals of this study were: (i) to estimate the transferability within the genus Citrus of SNPs discovered from Clementine BACend sequencing (BES), (ii) to estimate bias associated with the very narrow discovery panel, and (iii) to evaluate the usefulness of the Clementine-derived SNP markers for diversity analysis and comparative mapping studies between the different cultivated Citrus species.
\end{abstract}

Results: Fifty-four accessions covering the main Citrus species and 52 interspecific hybrids between pummelo and Clementine were genotyped on a GoldenGate array platform using 1,457 SNPs mined from Clementine BES and 37 SNPs identified between and within C. maxima, C. medica, C. reticulata and C. micrantha. Consistent results were obtained from 622 SNP loci. Of these markers, 116 displayed incomplete transferability primarily in C. medica, $C$. maxima and wild Citrus species. The two primary biases associated with the SNP mining in Clementine were an overestimation of the $C$. reticulata diversity and an underestimation of the interspecific differentiation. However, the genetic stratification of the gene pool was high, with very frequent significant linkage disequilibrium. Furthermore, the shared intraspecific polymorphism and accession heterozygosity were generally enough to perform interspecific comparative genetic mapping.

Conclusions: A set of 622 SNP markers providing consistent results was selected. Of the markers mined from Clementine, $80.5 \%$ were successfully transferred to the whole Citrus gene pool. Despite the ascertainment biases in relation to the Clementine origin, the SNP data confirm the important stratification of the gene pools around C. maxima, C. medica and C. reticulata as well as previous hypothesis on the origin of secondary species. The implemented SNP marker set will be very useful for comparative genetic mapping in Citrus and genetic association in C. reticulata.

\footnotetext{
* Correspondence: patrick.ollitrault@cirad.fr

${ }^{1}$ CIRAD, UMR AGAP, Avenue Agropolis, TA A-108/02, 34398 Montpellier,

Cedex 5, France

Full list of author information is available at the end of the article
} 


\section{Background}

Single-Nucleotide Polymorphisms (SNPs) are the most frequent type of variation found in DNA [1]. As EST databases and whole genome sequences grow in availability, SNPs have become the most abundant and powerful polymorphic codominant markers that can be selected all along the genome [2]. SNPs allow the implementation of very dense genetic linkage maps in animals and plants [3-5]. Moreover, SNPs are generally considered to have a high identity by descent rate, and thus, they are very useful for genetic association studies [6,7]. The actual array methodologies for the high throughput genotyping of SNPs are built upon the principle of measuring the relative signal strength of two expected alleles $[8,9]$ and require the use of oligonucleotides corresponding to the direct flanking regions of the SNPs. This should present some limitations for germplasm genetic studies. The primary limitation is that the revealed genetic organization of the genotyped germplasm is strongly dependent on the discovery panel [10-15]. This ascertainment bias is particularly noted when SNPs are selected from only one sequenced heterozygous genotype, as proposed in Vitis vitifera L. from the whole genome sequence of the cultivar 'Pinot Noir'[16]. Moreover, unexpected alleles may exist at any polymorphism. These unknown or 'null' alleles can interfere with accurate genotyping of the expected alleles, potentially impacting genetic studies in a negative manner [17]. The frequency of these alleles should increase when working with wider genetic distances between the genotyped samples and the discovery panel. A recent review [18] analyzed the importance of the discovery panel and SNP mining methods for genetic studies on plant and animals.

Citrus is the most extensively produced tree fruit crop in the world. Despite controversial Citrus classification (in this study, the Swingle and Reece [19] classification is used) most authors now agree on the origin of cultivated citrus species. Scora [20] and Barret and Rhodes [21], working with biochemical and morphological polymorphism, respectively, were the first to suggest that three main primary citrus species originated most of the cultivated citrus: $C$. medica L. (citrons), C. reticulata Blanco (mandarins) and C. maxima L. Osbeck (pummelos). Molecular marker studies (Isoenzyme [22]; RFLP [23]; RAPD and SCAR [24]; AFLP [25]; and SSRs $[26,27]$ generally support the role of these three taxa as ancestors of cultivated Citrus. Furthermore, these studies highlight the probable contribution of a fourth taxon, C. micrantha Wester, as the ancestor of limes $(C$. aurantifolia (Christm.) Swingle). All citrus species are fully sexually compatible, capable of producing fertile interspecific hybrids. Thus, they are all part of the same biological species and should probably be considered as separate races, rather than different species. Most modern cultivars have an interspecific origin [28]. All of the secondary species arising from hybridization among the primary species have been clonally propagated (facultative apomixis and horticultural practices), and as such, they present a generally high fixed heterozygosity. Clementine is such a hybrid, vegetatively propagated by grafting from the time it was selected as a chance offspring in a seedling of 'Mediterranean' mandarin (C. reticulata) one century ago. A haploid Clementine line has been chosen by the International Citrus Genomic Consortium (ICGC) to establish the reference Citrus whole genome sequence $[29,30]$. In the framework of the same international project, the diploid Clementine cv 'Nules' has been re-sequenced using new sequencing technologies (454, Roche). SNP density in Clementine has been previously estimated using BACend sequences (BES) to be close to $1 \mathrm{SNP} / \mathrm{Kb}$ [31]. As the Clementine haploid genome is estimated at $367 \mathrm{Mb}$ [32], this project is expected to deliver several hundred thousand SNPs all over the Citrus genome.

The primary goals of the present study were: (i) to assess the use by array genotyping and the transferability of SNPs discovered from the heterozygous Clementine genome within the Citrus genus; (ii) to compare the genetic structure revealed by SNPs heterozygous in Clementine with the structure displayed by SNPs found at the genus level and homozygous in Clementine; (iii) to investigate hypotheses concerning the origin of some secondary species and important cultivars; and (iv) to estimate the usefulness of the Clementine-derived SNP markers for comparative mapping studies between the various cultivated Citrus species. For these purposes, 54 Citrus accessions and 52 interspecific hybrids between 'Chandler' pummelo and 'Nules' Clementine $(\mathrm{CxN})$ were genotyped on a GoldenGate array platform (Illumina) using 1457 SNPs mined from Clementine cv 'Nules' BES [31] and 37 SNPs mined from between and within $C$. maxima, C. medica, C. reticulata and C. micrantha.

\section{Results}

Design of the Citrus Illumina GoldenGate SNP set SNP selection from Clementine BES

Among the 6,617 SNPs mined in silico using the POLYBAYES software on $6.14 \mathrm{Mb}$ of assembled sequences from $\mathrm{BES}$, transitions $([\mathrm{A} / \mathrm{G}]+[\mathrm{C} / \mathrm{T}])$ represented the most abundant changes $(3,546 ; 53.6 \%)$. These were followed by transversions $([\mathrm{A} / \mathrm{C}]+[\mathrm{G} / \mathrm{T}], 2,162 ; 32.7 \%)$ and InDels $(909 ; 13.7 \%)$. According to their probability robustness value, 4,904 transition and transversion SNPs were selected to be tested for their potential technical inclusion on the GoldenGate array. Based on the flanking sequences and absence/presence of additional known SNPs in the vicinity, 2,185 sequences generated 
a SNP_score greater than 0.6, which was considered the threshold for good marker designability. A total of 768 additional markers exhibited SNP_scores between 0.4 and 0.6 and were associated with a moderate success rate for the marker. Finally, among these 2,953 potential markers, 1,457 SNPs (1,434 with an SNP_score > 0.6 and 23 with an SNP_score between 0.4 and 0.6) were selected for the GoldenGate assay. This selection was based on the SNP distribution on the different BACend contigs and the SNP inclusion or vicinity to coding regions (additional file 1). Respectively, $60.6 \%$ were transitions $([\mathrm{A} / \mathrm{G}]+[\mathrm{C} / \mathrm{T}]=883)$ and $39.4 \%$ were transversions $([\mathrm{A} / \mathrm{C}]+[\mathrm{G} / \mathrm{T}]=311 ;[\mathrm{A} / \mathrm{T}]=167 ;[\mathrm{G} / \mathrm{C}]=96)$.

\section{SNP selection from the amplified fragments of gene}

\section{sequences in the Citrus genus}

A total of $6.953 \mathrm{~kb}$ were sequenced (Sanger) following the targeted amplification of 10 gene fragments for each of the seven genotypes of the four primary taxa of cultivated species. Two hundred and four SNPs were identified (29.3 SNPs/kb; additional file 2). The designability for the GoldenGate assay was tested using 121 of the identified SNPs. Respectively, 45 and 15 displayed a SNP_score over 0.6 and between 0.4 and 0.6 . Thirty seven SNPs were ultimately included in the GoldenGate assay (additional file 1). Of these, 67.5\% represented transitions and $32.5 \%$ represented transversions.

\section{Polymorphism and allele call for the different SNPs; selection and classification of valid SNPs}

For all SNPs, the genotyping was visually confirmed, taking advantage of the distribution of the $\mathrm{CxN}$ progenies relatively to 'Nules' clementine and 'Chandler' pummelo positions. The SNPs were assigned to different categories based on the quality of the polymorphism detected, the detection of null alleles, and the type of segregation observed for 'Nules' Clementine in the $\mathrm{CxN}$ progeny (additional file 1 ). The first category (C1) consisted of 230 markers exhibiting very low technical quality which did not allow for clustering. Among the other categories:

- 608 (C2) displayed the expected segregation for clementine in the $\mathrm{CxN}$ progeny. However, for 80 of these loci the clustering between the three classes of genotypes was not totally clear, leading to missing data.

- 85 (C3) with validated heterozygosity in Clementine presented an unexpected segregation, revealing heterozygous (Figure 1a) or homozygous (Figure 1b) null alleles in 'Chandler' pummelo or some others germplasm accessions.

- 28 (C4) displayed segregation of the $\mathrm{CxN}$ progeny, supporting a heterozygous null allele in Clementine (ure 1c).
- For the four last categories, no segregation from Clementine was observed in the $\mathrm{CxN}$ progeny. Seventy five markers were polymorphic on the whole germplasm sample, displaying homozygous and heterozygous genotypes (C5; Figure 1d); however, 25 were of low quality. Consistent interspecific polymorphisms for a null allele were observed for 32 markers (C6; Figure 1e). For 62 markers, no polymorphism was observed within the sample. However, the cluster position corresponded to an equivalent signal of the two alleles (heterozygouslike, C7, Figure 1f), suggesting possible loci duplication. The last category of 374 markers (C8) consisted of loci with no observed polymorphism. Of the 683 polymorphic loci without null alleles (WONA; C2 $+\mathrm{C} 5$ ) and the 145 loci with null alleles (WNA; C3 + C4+C6), respectively 506 and 116 markers displaying the clearest differentiation between genotypic classes (unambiguous assigning of genotypes with less than $5 \%$ missing data) were selected for further analysis. For the selected WNA and WONA loci, the transition/transversion rate was 59.8/41.1 and 59.5/ 40.5 , respectively. These values are very close to the rate initially observed for the mined SNPs. Respectively, 480 and 26 of the selected WONA loci were from BES and gene sequencing, while all markers with null allele were from BES.

To validate the genotyping data, 24 of the 54 Citrus accessions that were genotyped with the GoldenGate array were sequenced (Sanger) for 15 SNPs from five genes. Among the 360 genotype $\times$ SNP data, 357 (99.2\%) were in agreement with the GoldenGate genotyping and Sanger sequences. In two cases (PSY-C-246 for 'Eureka' lemon and LCY2-P-75 for 'Sunki' mandarin), the GoldenGate genotyping concluded heterozygosity, while the Sanger sequencing inferred homozygosity. The opposite case was obtained with PSY-C-497 in Mexican lime.

The origin of the unexpected polymorphisms displayed by several SNP markers from the Clementine BES, such as null alleles, no heterozygosity for Clementine and 'fixed heterozygosity', was analyzed using Sanger sequencing of the amplicons of four accessions: 'Nules' Clementine, haploid Clementine, 'Chandler' pummelo and Corsican citron.

For 7 loci (CiC0002-01; CiC0049-02; CiC0063-12; CiC0074-09; CiC0091-09; CiC0113-01; CiC2553-04) genotyped homozygous for Clementine in Golden Gate, despite previously being labeled as heterozygous based on BES sequencing, the homozygosity was confirmed by Sanger sequencing. For the analyzed locus of apparent fixed heterozygosity in the PxC progeny (CiC4252-10), the haploid Clementine line also displayed a 


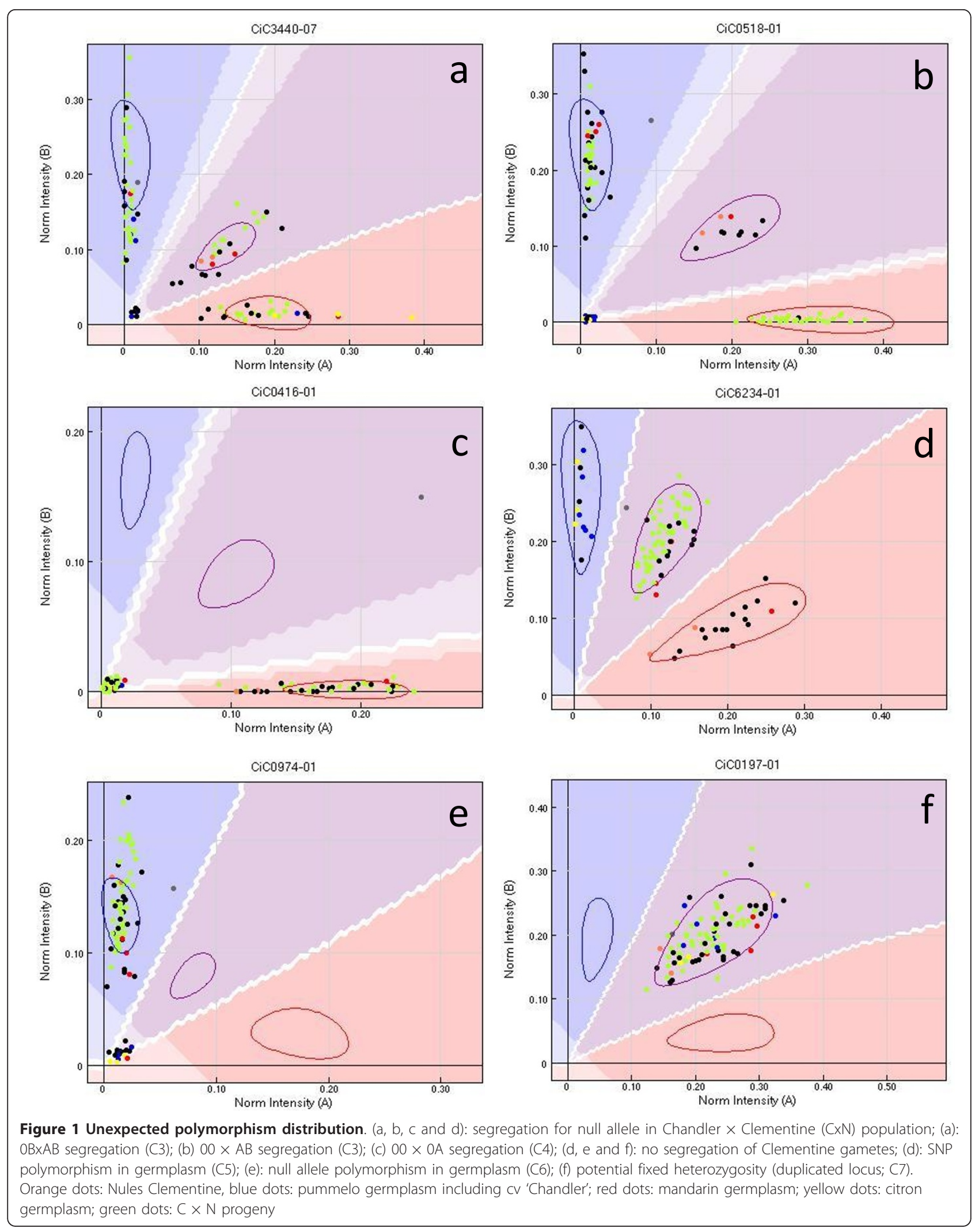


heterozygous-like pattern, thus confirming the hypothesis of a duplicated locus. For the loci with null alleles, fragment amplification was observed for all of the genotypes. Additional SNPs in the GoldenGate primer area were observed for $\mathrm{CiC} 3064-07$ and $\mathrm{CiC} 3275-02$ in 'Chandler' pummelo and Corsican citron. However, for another marker of this class (CiC2151-02), no polymorphisms or explanation for the null alleles was found.

\section{Citrus Germplasm diversity for markers without null alleles (WONA loci)}

Population genetic parameters: detailed data for the 506 markers are given in the additional file 3 and summarized in table 1 . The proportion of polymorphic loci was very high within $C$. reticulata $(90.7 \%)$ while the lower polymorphic loci percentages were observed for $C$. medica (15.4\%) and C. maxima (26.9\%). The average Nei diversity (He) for all of the loci in the entire sample was 0.34 , and this varied for individual locus between 0.05 and 0.50 (additional file 3). Thirty-five loci with rare alleles (frequency inferior to 0.05 - ie $\mathrm{He}<0.095$ ) were observed. All genotypes were differentiated with the exception of the two C. aurantium accessions. The data from the loci determined to be heterozygous (CHet; 476 loci) or homozygous (CHom; 30 loci) in Clementine (table 2) were analyzed separately. Significant differences were observed for the two classes of markers. The level of diversity revealed within $C$. reticulata was strongly reduced for $\mathrm{CHom}$ compared with $\mathrm{CHet}$, with respect to the rates of polymorphic loci $(56.7 \% / 92.9 \%)$, Nei diversity (He: $(0.16 / 0.29)$, and observed heterozygosity $(0.17 / 0.34)$. A similar reduction of polymorphism was observed for $C$. sinensis while the average diversity described by the same parameters was significantly higher for $\mathrm{CHom}$ for three other secondary species $(C$.

Table 1 Genetic diversity parameters for all loci without null allele (WONA; 506).

\begin{tabular}{lccccc}
\hline & $\begin{array}{c}\text { \% L. } \\
\text { P. }\end{array}$ & MLGs & Ho & He & F \\
\hline C. maxima (10) & 26.88 & 10 & $0.063+/-0.012$ & $0.063+/-0.011$ & \\
C. medica (5) & 15.42 & 5 & $0.089+/-0.021$ & $0.057+/-0.012$ & \\
C. reticulata (12) & 90.71 & 12 & $0.327+/-0.019$ & $0.279+/-0.013$ & \\
C. aurantifolia (4) & 54.55 & 4 & $0.217+/-0.021$ & $0.186+/-0.015$ & \\
C. aurantium (2) & 54.74 & 1 & $0.547+/-0.043$ & $0.273+/-0.021$ & \\
C. limon (7) & 68.97 & 7 & $0.364+/-0.030$ & $0.267+/-0.017$ & \\
C. paradisi (2) & 41.70 & 2 & $0.416+/-0.042$ & $0.208+/-0.021$ & \\
C.sinensis (4) & 65.61 & 4 & $0.653+/-0.041$ & $0.327+/-0.020$ & \\
Wild Citrus (5) & 47.23 & 5 & $0.118+/-0.012$ & $0.150+/-0.013$ & \\
All samples (54) & 100.00 & 53 & $0.278+/-0.010$ & $0.336+/-0.011$ & 0.127 \\
& & & & & $+/-0.019$ \\
\hline
\end{tabular}

\%LP: percentage of polymorphic loci, MLGs: number of multilocus genotypes differentiated; Ho: observed heterozygosity; He: Nei diversity; F: Wright fixation index
Table 2 Comparison of diversity parameters for loci without null allele (WONA) homozygous (CHom; 30) or heterozygous (Chet; 476) in Clementine.

\begin{tabular}{|c|c|c|c|c|c|}
\hline & & $\begin{array}{c}\% \text { L. } \\
\text { P. }\end{array}$ & Ho & $\mathrm{He}$ & $\mathrm{F}$ \\
\hline \multirow[t]{2}{*}{ C. maxima (10) } & $\mathrm{CHom}$ & 16.67 & $0.036+/-0.031$ & $0.042+/-0.039$ & \\
\hline & CHet & 27.52 & $0.065+/-0.012$ & $0.064+/-0.011$ & \\
\hline \multirow[t]{2}{*}{ C. medica (5) } & CHom & 20.00 & $0.061+/-0.057$ & $0.047+/-0.038$ & \\
\hline & CHet & 15.13 & $0.091+/-0.022$ & $0.057+/-0.013$ & \\
\hline \multirow[t]{2}{*}{ C. reticulata (12) } & CHom & 56.67 & $0.166+/-0.075$ & $0.161+/-0.062$ & \\
\hline & CHet & 92.86 & $0.337+/-0.019$ & $0.286+/-0.013$ & \\
\hline \multirow[t]{2}{*}{ C. aurantifolia (4) } & $\mathrm{CHom}$ & 66.67 & $0.366+/-0.128$ & $0.295+/-0.064$ & \\
\hline & CHet & 53.78 & $0.208+/-0.021$ & $0.179+/-0.015$ & \\
\hline \multirow[t]{2}{*}{ C. aurantium (2) } & CHom & 43.33 & $0.433+/-0.180$ & $0.216+/-0.090$ & \\
\hline & CHet & 55.46 & $0.554+/-0.044$ & $0.277+/-0.022$ & \\
\hline \multirow[t]{2}{*}{ C. limon (7) } & CHom & 80.00 & $0.579+/-0.136$ & $0.378+/-0.068$ & \\
\hline & CHet & 68.28 & $0.351+/-0.031$ & $0.259+/-0.017$ & \\
\hline \multirow[t]{2}{*}{ C. paradisi (2) } & CHom & 63.33 & $0.633+/-0.175$ & $0.316+/-0.087$ & \\
\hline & CHet & 40.34 & $0.402+/-0.044$ & $0.201+/-0.022$ & \\
\hline \multirow[t]{2}{*}{ C.sinensis (4) } & CHom & 36.67 & $0.358+/-0.172$ & $0.182+/-0.087$ & \\
\hline & CHet & 67.44 & $0.671+/-0.042$ & $0.336+/-0.021$ & \\
\hline \multirow[t]{2}{*}{ Wild Citrus (5) } & CHom & 26.67 & $0.068+/-0.044$ & $0.200+/-0.064$ & \\
\hline & CHet & 48.53 & $0.121+/-0.013$ & $0.147+/-0.013$ & \\
\hline \multirow[t]{2}{*}{ All samples (54) } & CHom & 100.00 & $0.239+/-0.035$ & $0.403+/-0.024$ & $\begin{array}{c}0.405 \\
+/-0.081\end{array}$ \\
\hline & CHet & 100.00 & $0.280+/-0.010$ & $0.331+/-0.012$ & $\begin{array}{r}0.109 \\
+/-0.019\end{array}$ \\
\hline
\end{tabular}

\%LP: percentage of polymorphic loci, Ho: observed heterozygosity; He: Nei diversity; F: Wright fixation index

limon, C. aurantifolia and C. paradisi). No significant differences were observed for C. medica and C. maxima. For wild Citrus, fewer markers were polymorphic when homozygous in Clementine. Throughout the entire sample, the average diversity was slightly higher for the CHom class than with the CHet class $(0.403 / 0.331)$ with a much stronger population stratification (0.405 and $0.109 \mathrm{~F}$ values, respectively).

Structuration between basic taxa: the analysis of the Fstat parameter in considering the accessions of $C$. $\max$ ima, C. medica and C. reticulata confirmed the higher differentiation between species with $\mathrm{CHom}$ markers (table 3). Indeed, when the Fis value indicated no significant deviance from HWE with the two classes of markers, the significant Fit value (mainly due to between species differentiation with a high positive Fst value) is close to double for the CHom markers $(0.80 / 0.40$ for Fit and $0.80 / 0.45$ for Fst). Very similar Fst values are estimated when considering only the differentiation between C. maxima and C. reticulata (table 3 ). NJ representations with the two set of markers (Figure 2) clearly shows an increase in inter-specific differentiation with the CHom marker set compared to the CHet set. Particularly, with the CHom markers, C. medica is 
Table 3 Population organization parameters (Fstat) between and within the three basic taxa and differentiation between C.maxima and C. reticulata (Fst) evaluated with loci heterozygous (476) or Homozygous in Clementine (30).

\begin{tabular}{|c|c|c|c|c|}
\hline & \multicolumn{3}{|c|}{$\begin{array}{c}3 \text { basic taxa } \\
\text { (C. maxima, C. medica, C. reticulata) }\end{array}$} & \multirow{2}{*}{$\begin{array}{c}\text { C. maxima } \\
\text { /C. reticulata } \\
\text { Fst }\end{array}$} \\
\hline & Fis & Fit & Fst & \\
\hline All loci & $-0.09+/-0.02$ & $0.42+/-0.03$ & $0.47+/-0.03$ & $0.46+/-0.03$ \\
\hline Het. Cle. & $-0.10+/-0.02$ & $0.40+/-0.03$ & $0.45+/-0.03$ & $0.45+/-0.03$ \\
\hline Hom. Cle. & $0.012+/-0.14$ & $0.80+/-0.10$ & $0.80+/-0.08$ & $0.70+/-0.11$ \\
\hline
\end{tabular}

strongly differentiated from the two other species. Moreover, within $C$. reticulata, the dissimilarities between 'Cleopatra', Sun Chu Sha', 'Mediterranean', 'Imperial', 'Ponkan', 'Fushu' and 'Dancy' genotypes appears significantly smaller with CHom than with CHet markers.

Neighbor joining (NJ) and principal component analysis (PCA) of the whole germplasm sample: $\mathrm{NJ}$ trees are not well adapted to describing the genetic relationships of hybrid structure with the parental gene pool. However, NJ trees allow a global synthetic representation of dissimilarities between genotypes. In factorial analyses such principal component analysis, the hybrid positions between the parents is very clear in the axes that differentiate the parental genotypes. However, representation in a two- or three-axes space may result in a biased view of the global similarities between genotypes. The two representation types (NJA and PCA) are thus complementary when analyzing the genetic relationships and potential parentage of hybrid genotypes. The genetic organization around $C$. reticulata, C. medica and $C$. maxima appears very clearly both with the PCA (Figure 3a) and NJA (Figure 4). Forty-eight percent of the entire diversity is represented by the first two axis of PCA. The first axis discriminates $C$. reticulata from the other species while the second axis separates $C$. medica and C. maxima. Very few within species differentiations are observed for the accessions of $C$. aurantium, $C$. sinensis and C. paradise. By contrast, C. aurantifolia and C. limon display more intra-specific polymorphism. Two accessions of $C$. aurantifolia, 'Alemow' and 'Mexican lime', exhibit an intermediate position between C. med$i c a$ and a papeda cluster including $C$. ichangensis, $C$. micrantha and C. hystrix. The other two C. aurantifolia accessions (Calabria and Palestine sweet lime) are more related to the $C$. limon accessions. The C. limon accessions are subdivided into three close clusters: Meyer lemon is the more isolated, while 'Eureka', 'Lisbon' and 'Marrakech lime' are clustered to one side and 'Rangpur' lime, 'Rough lemon' and 'Volkamer' lemon cluster in the other side. This last cluster presents higher coordinates in the second axis of the PCA(a) and lower coordinates in the first axis, compared to the first lemon cluster. This suggests a higher contribution of $C$. reticulata and very few or no $C$. maxima contribution. Interestingly, a sub cluster of acidic mandarin ('Cleopatra', 'Sunki', 'Depressa' and 'Sun Chu Sha') also present higher coordinates in the second axis relative to the other C. reticulata accessions. Sweet and sour oranges are strongly differentiated by the second axis. Clementine is very close to sweet orange in this axis and is intermediary between Mediterranean mandarin and sweet orange in the first axis. To analyze the contribution of $C$. maxima and $C$. reticulata to the genome of their supposed deriving secondary species (C. sinensis, $C$. aurantium and $C$. paradisi), a second PCA was generated using only $C$. maxima and C. reticulata as active individuals to define the new axes (Figure 3b). Of the total diversity, 52.1\% was supported by the first axis opposing the two species. The contribution of the SNP loci to the definition of this axis (estimated by the $\cos ^{2}$ of the coordinate in the new components) presents a very high correlation with the Fst value for the C. maxima/C. reticulata differentiation $\left(r^{2}=0.948\right.$, additional file 4 , figure S1). This confirms the validity of this axis for the synthesis of the relative contribution of the two basic species to the secondary ones. C. sinensis and C. aurantium display similar positions in this axis at a closer distance to the $C$. reticulata gene pool than the C. maxima one. C. paradisi has an intermediary position between these two secondary species and C. maxima.

Linkage disequilibrium in the germplasm and segregating population: 472 of the 476 WONA markers heterozygous for Clementine have been successfully genotyped (less than $5 \%$ missing data) in the 'Chandler' pummelo $\times$ 'Nules' Clementine progeny $(\mathrm{CxN})$. A comparative analysis of the LD within the Citrus germplasm sample and within the $\mathrm{CxN}$ segregating population was done for all of the pairs of the 472 markers. The average LD (estimated by $\mathrm{r}^{2}$ ) was 0.152 and 0.060 for germplasm and $\mathrm{CxN}$, respectively. $\mathrm{A} \mathrm{r}^{2}>0.2$ is generally considered to be a threshold for significant LD between marker pairs. Using this criteria, $27 \%$ and $6 \%$ of the locus pairs displayed significant LD within the germplasm and $\mathrm{CxN}$ populations, respectively. The proportion of significant LD was estimated using the exact test p-value at 5\% and $1 \%$ thresholds. Regardless of the parameter used, the proportion of significant LD was much higher within the germplasm sample than with the segregating $\mathrm{CxN}$ population (table 4). Upon analyzing the co-distribution of LD for the germplasm and segregating populations (Additional file 4, figure S2), an important proportion of significant LD were observed within the germplasm population for the loci pairs with $r^{2}<0.1$ in the segregating $\mathrm{CxN}$ population. 


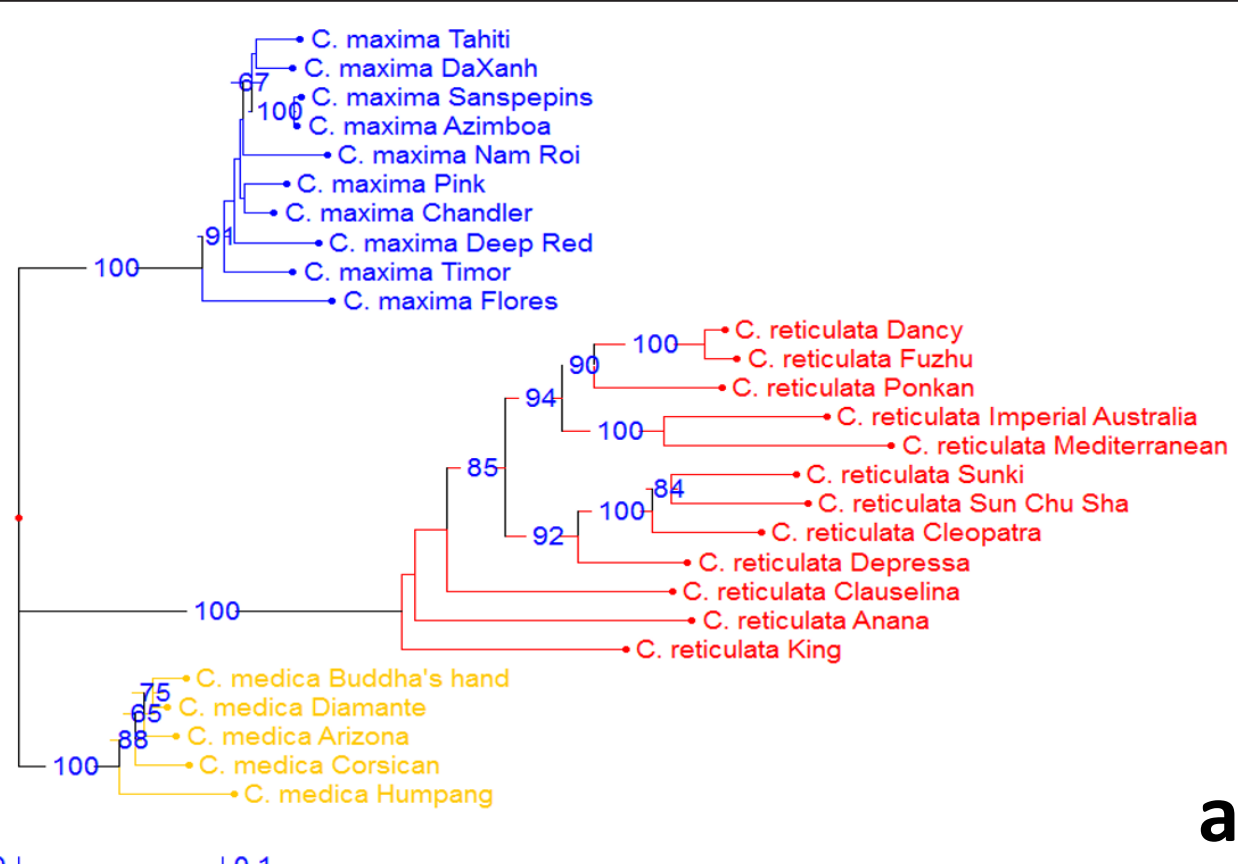

$0 \longmapsto 0.1$

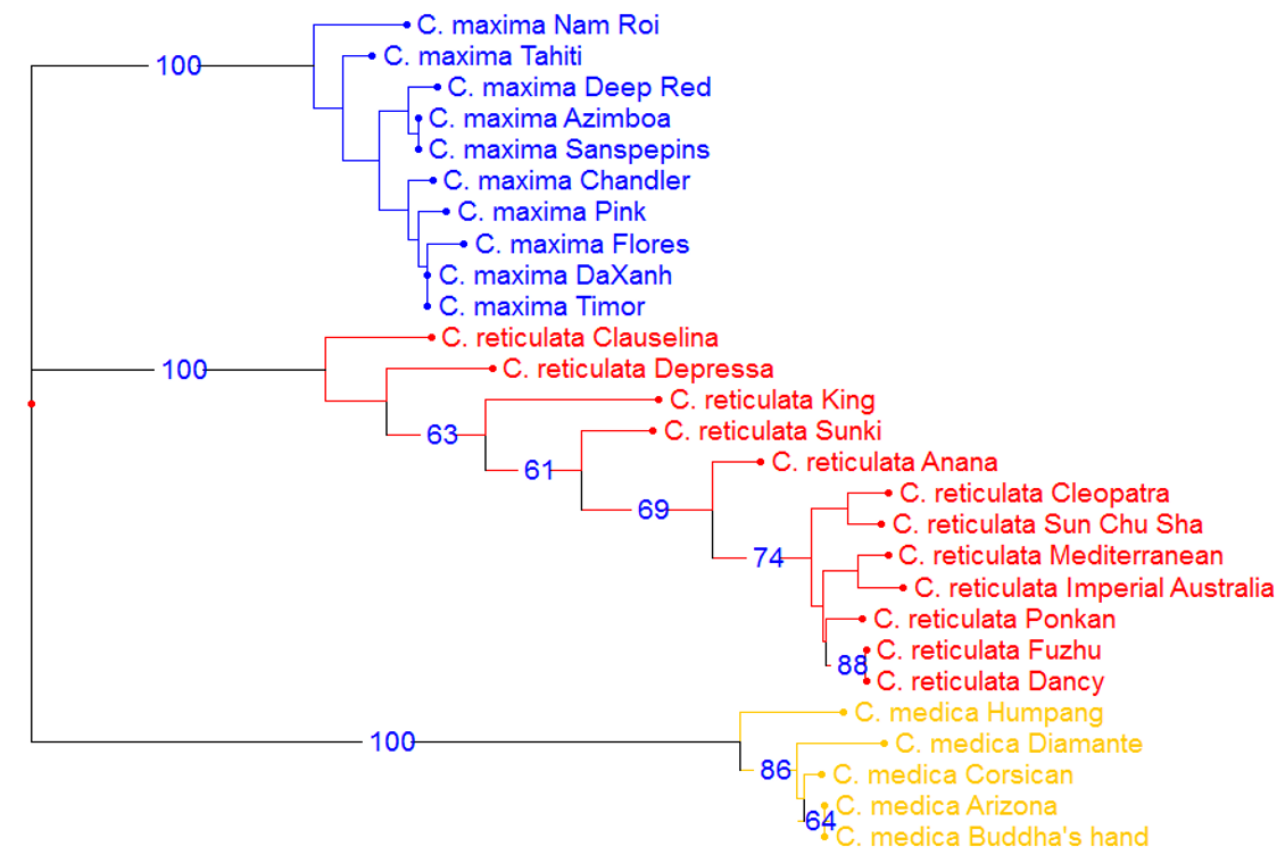

$0 \longmapsto 0.1$ Dissimilarity index scale

b

Figure 2 Cluster analysis (Neighbor Joining) of the accessions of C. reticulata, C. maxima and C. medica based on loci without null allele (WONA). Loci heterozygous in Clementine (CHet; 476); b) Loci homozygous in Clementine (CHom; 30). Numbers near nodes are bootstrap values based on 1000 resamplings (only value $>60 \%$ are indicated). 

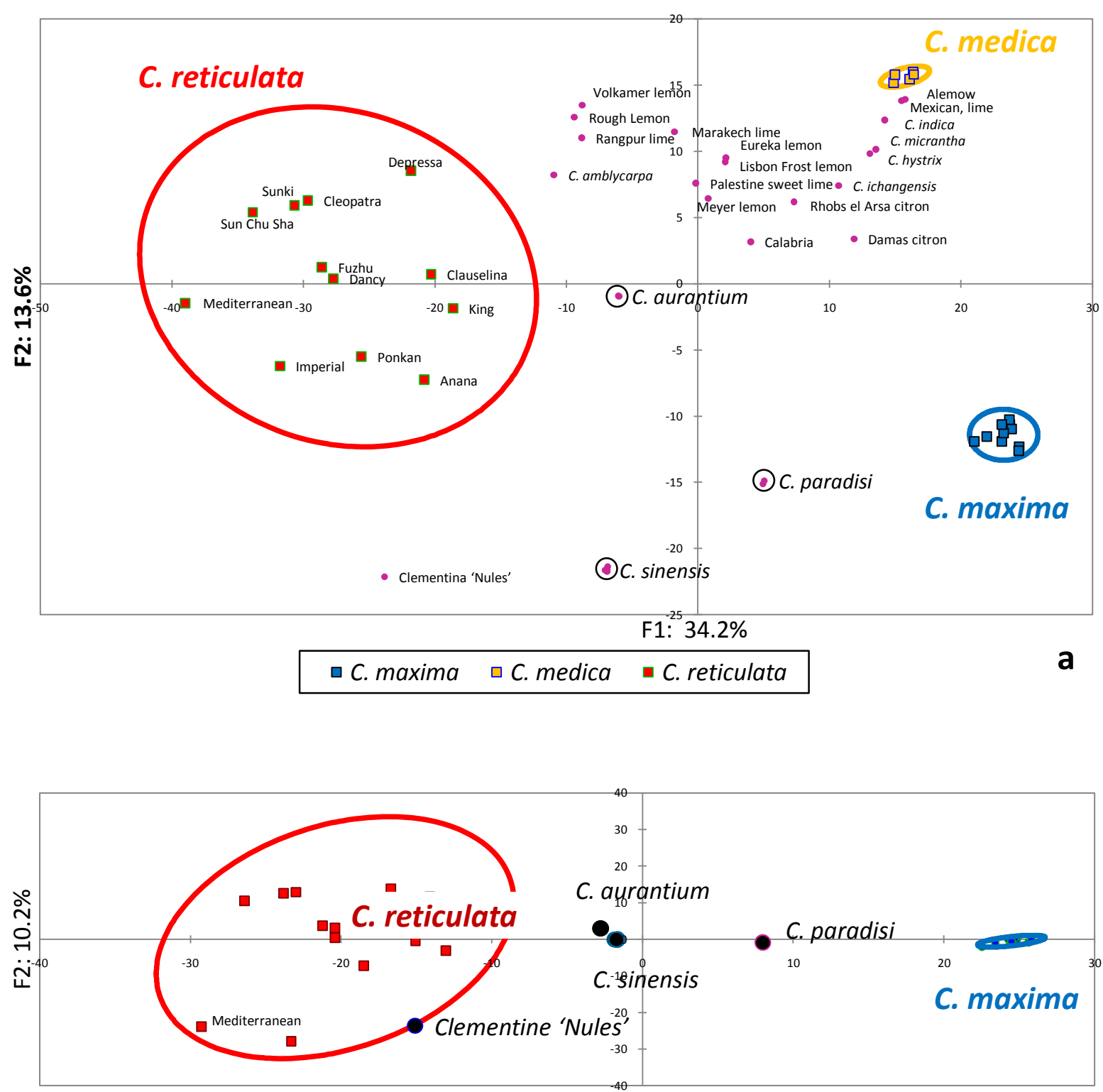

F1: $52.1 \%$

Figure 3 Principal component analysis from all loci without null alleles (506 loci). a) Axes 1/2 with all individual active. b) Positions of secondary species in the axis 1 of an ACP established from only C. reticulata and C. maxima as active accessions.

\section{Citrus Germplasm diversity displayed by markers with} null alleles (WNA loci)

Detailed data for all WNA markers are listed in additional file 5 and summarized in table 5. Among the 116 markers with null alleles, 88, 82, 31 and 92 display null alleles for at least one accession within C. maxima, $C$. medica, C. reticulata and wild Citrus, respectively. Interestingly, for $C$. reticulata, nine of the 31 considered markers exhibit null allele for only one accession. The frequency of genotypes that are homozygous for the null allele is very high in C. medica (0.641), C. maxima
(0.609), wild Citrus (0.474) and C. aurantifolia (0.405), but low in $C$. reticulata $(0.111)$ and $C$. sinensis (0.086).

The within species discrimination of accessions obtained with the whole set of WNA markers is respectable, with the exception of $C$. sinensis and $C$. paradisi. $C$. reticulata, the most polymorphic species, displayed a genotypic diversity of 0.361 and an observed heterozygosity of 0.234 . The observed heterozygosity of all of the secondary species is strongly reduced when compared with the WONA loci, suggesting frequent but not observed heterozygous null alleles. 


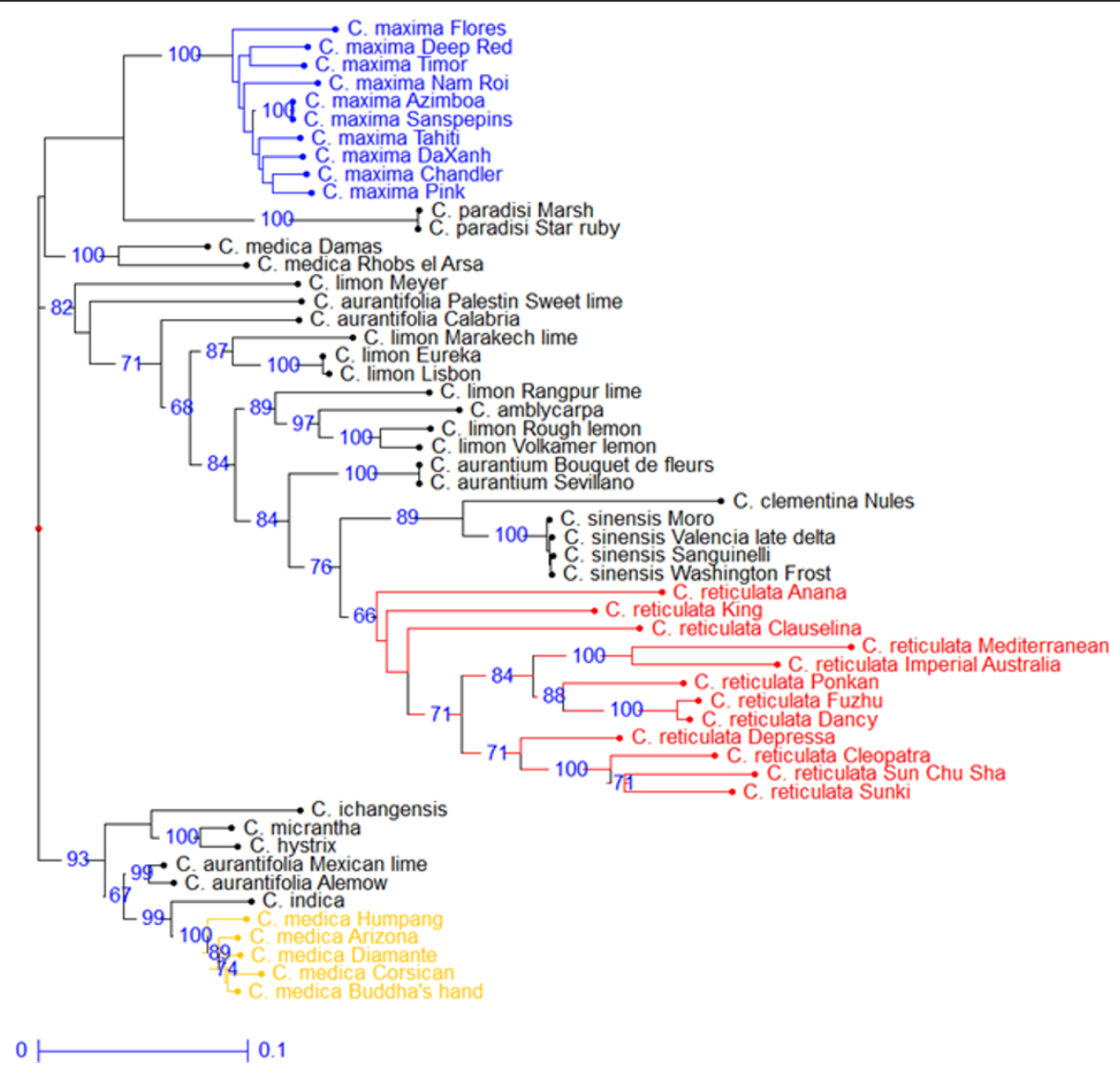

Figure 4 Neighbor Joining Analysis based on all loci without null alleles (WONA; 506 loci). Numbers near nodes are bootstrap values based on 1000 resamplings (only value $>60 \%$ are indicated).

The differentiation of the three basic species (C. maxima, C. medica and C. reticulata) was confirmed by the NJA based on WNA loci (Figure 5). However, the global picture displayed by this analysis varies significantly from the previous one without null alleles (Figure 4). Indeed, C. maxima, C. medica, most wild Citrus species (except $C$. amblicarpa), as well as two C. aurantifolia accessions (Mexican lime and Alemow) are strongly clustered. The numerous loci sharing null alleles between C. medica, C. maxima and some wild species may explain this strong clustering as well as the position of supposed hybrids between these taxa (Mexican lime and Alemow). Moreover, C. sinensis, C. paradisi and

Table 4 LD between 472 SNP loci within the Chandler $x$ Nules progeny and germplasm samples.

\begin{tabular}{lcccc}
\hline & $\begin{array}{c}\text { Average } \\
\mathbf{r}^{\mathbf{2}}\end{array}$ & $\mathbf{r}^{\mathbf{2}}>\mathbf{0 . 2}$ & $\begin{array}{c}\text { pvalue }< \\
\mathbf{5 \%}\end{array}$ & $\begin{array}{c}\text { pvalue }< \\
\mathbf{1 \%}\end{array}$ \\
\hline Chandler $\times$ & 0.060 & $7015(6.31 \%)$ & 12962 & $8488(7.64 \%)$ \\
Nules & & & $(11.66 \%)$ & \\
Germplasm & 0.152 & 29813 & 65758 & 49169 \\
& & $(26.82 \%)$ & $(59.15 \%)$ & $(44.23 \%)$ \\
\hline
\end{tabular}

several C. limon and C. aurantifolia accessions are integrated within the $C$. reticulata cluster. These secondary species are suspected to be hybrids between $C$. reticulata and C. maxima and/or C. medica. Due to the recessive nature of null alleles, these secondary species

\section{Table 5 Diversity parameters for loci with null alleles} (WNA)

\begin{tabular}{lccccc}
\hline & \% L. P. & MLGs & GD & Null & Ho \\
\hline C. maxima (10) & 41.38 & 10 & $0.145+/-0.036$ & 0.609 & 0.037 \\
C. medica (5) & 17.24 & 5 & $0.060+/-0.025$ & 0.641 & 0.041 \\
C. reticulata (12) & 80.17 & 12 & $0.361+/-0.040$ & 0.111 & 0.234 \\
C. aurantifolia (4) & 70.69 & 4 & $0.308+/-0.038$ & 0.405 & 0.045 \\
C. aurantium (2) & 15.52 & 2 & $0.004+/-0.008$ & 0.103 & 0.151 \\
C. limon (7) & 73.27 & 6 & $0.317+/-0.041$ & 0.190 & 0.086 \\
C. paradisi (2) & 8.62 & 1 & $0+/-0$ & 0.224 & 0.086 \\
C. sinensis (4) & 24.13 & 1 & $0 /-0$ & 0.086 & 0.241 \\
Wild Citrus (5) & 76.72 & 5 & $0.329+/-0.037$ & 0.474 & 0.036 \\
All samples (54) & 100 & 47 & $0.546+/-0.021$ & 0.326 & 0.121 \\
\hline
\end{tabular}

\%LP: percentage of polymorphic loci, MLGs: number of multilocus genotypes differentiated; GD: genotypic diversity; Null: average proportion of

Homozygous for null allele; Ho: average proportion of observed heterozygous (2 different bases). 


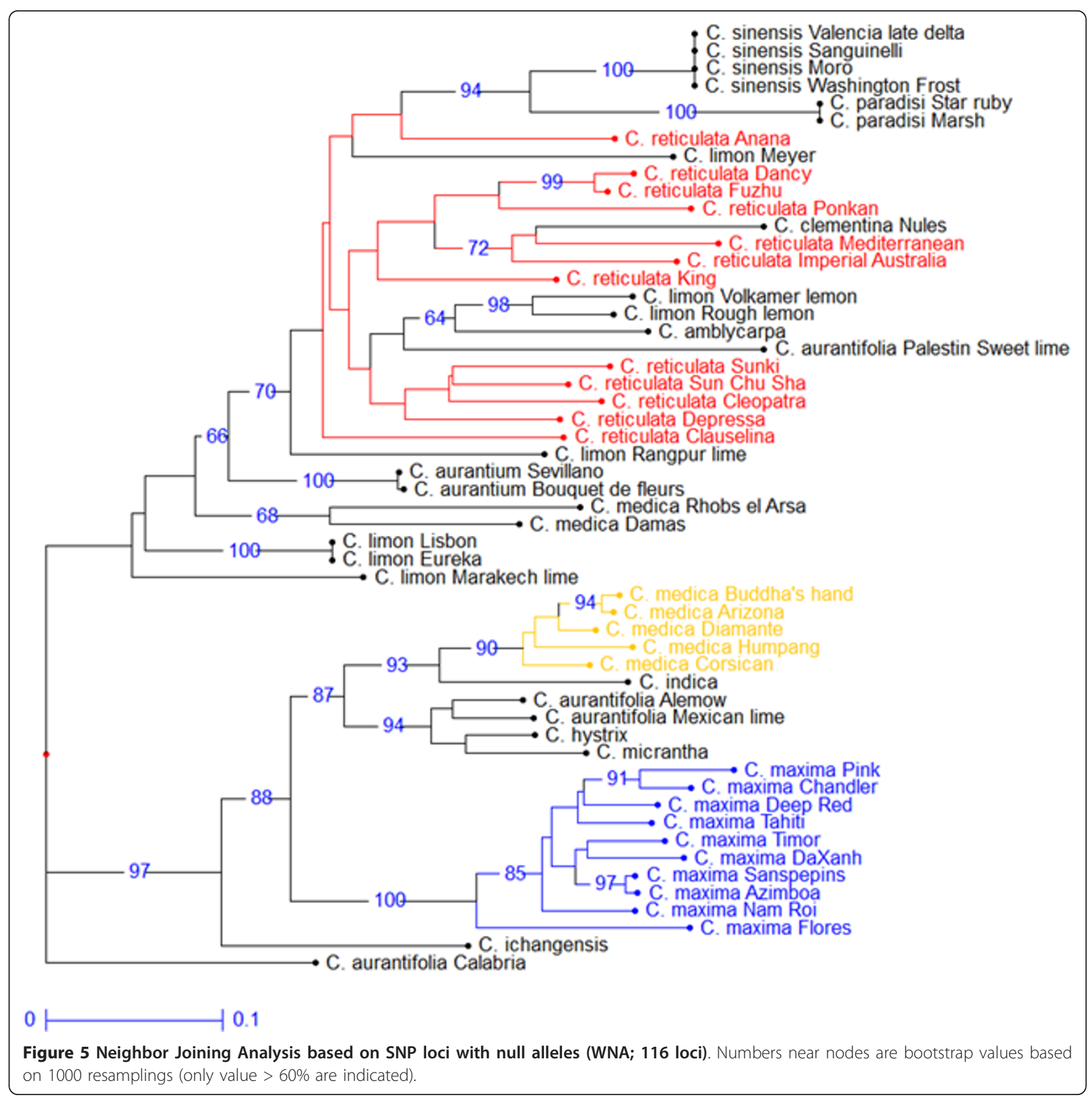

appear artificially closer to their C. reticulata parent because of the lower frequency of null alleles in this gene-pool compared with the other ancestral species. An interesting point is that the recessive nature of a high proportion of alleles from the other species allows the approximation of the sub gene pool within C. reticulata at the origin of some interspecific hybrids. Clementine is clustered with 'Mediterranean' mandarin. The parentage of sweet orange and grapefruit is also clearly revealed. Volkamer and Rough lemons, as well as Palestine Sweet lime, are clustered with a group of acidic mandarins (Sunki, Sun Chu Cha, Cleopatra and Depressa). C. amblycarpa is also associated with this cluster.

\section{Markers for interspecific comparative genetic mapping}

The number of loci that could be potentially mapped was estimated as the number of polymorphic loci within each species. For the WNA markers, the SNPs as well as the null allele polymorphisms were considered. As Clementine was at the origin of most of the selected SNPs markers, 'Nules' Clementine was logically the 
cultivar allowing the most complete genetic mapping with a total of 567 mappable markers (table 6). At the intraspecific level, the usefulness of the selected SNP loci is high for C. reticulata (554), C. limon (460), C. aurantifolia (377) and C. sinensis (361), moderate for $C$. paradisi (221) and C. maxima (189), low for C. medica (99), and very low for C. micrantha (17). Compared to the proportion for WONA loci, the proportion of useful WNA loci appears low for C. paradisi (10/116), C. sinensis (29/116) and C. aurantium (18/116), but very high for C. reticulata (93/116), C. limon (85/116) and C. aurantifolia (82/116). The highest number of loci for comparative mapping using WONA markers was observed for C. reticulata/C. limon (350), followed by $C$. reticulata/C. sinensis (301). For WNA markers, the higher values were found for C. aurantifolia/C. limon and $C$. aurantifolia/C. reticulata (63 markers for both interspecific comparative mapping).

\section{Discussion}

SNP mining in Clementine and the unexpected segregations in Chandler $\times$ Clementine progeny

Among the 6,617 SNPs mined in silico, 1,457 markers were selected for the GoldenGate assay based on their distribution on the different BACend contigs, as well as their inclusion in or their vicinity to the coding region. Thirty seven SNP loci found in 10 candidate genes were added for the analysis. Low technical quality was displayed by 230 markers, preventing any clustering. A total of 693 markers presented segregations that conformed to Clementine heterozygosity $(\mathrm{C} 2+\mathrm{C} 3)$. The 'Chandler' $\times$ Clementine $(\mathrm{CxN})$ progeny revealed heterozygous null alleles in Clementine for 28 markers (C4). Moreover, 481 markers appeared to be homozygous for Clementine $(\mathrm{C} 5+\mathrm{C} 6+\mathrm{C} 8)$ while 472 of them were supposed to be heterozygous from the Clementine BACend sequencing [31]. This homozygosity was confirmed using Sanger sequencing for the seven tested markers. SNP analysis on BAC ends was carried out by analyzing nucleotide variation within assembled reads in one contig, each reading originated from different $E$. coli clones. A base miscall in one of the BAC end reads, or even a mutation introduced by the $\mathrm{E}$. coli replication machinery in the BAC sequence, could create a false SNP that is not present in the genomic sequence, as it was confirmed by direct Sanger sequencing of the genomic DNA amplified by PCR. For 62 markers, potential locus duplication (C7) was suspected and confirmed for the tested locus by the heterozygous profile for the haploid Clementine line selected for whole genome sequencing [29]. Moerover multiple blasts in the reference citrus whole genome sequence (http://www.phytozome.net/ clementine.php) of the corresponding sequences comfort this hypothesis (data not shown). Five hundred ninety six of the SNPs mined in Clementine BES and 26 from targeted gene sequencing were finally selected for genetic analysis. The validation of the SNP genotyping for 15 SNPs $\times 24$ genotypes was conducted by Sanger sequencing and a validity rate of $99.2 \%$ was obtained.

\section{GoldenGate SNP marker transferability and loci with null allele usefulness}

Marker transferability was estimated by null allele identification and dispersion in the gene pool. The null alleles may result from unexpected polymorphisms affecting the allele amplification/hybridization on the GoldenGate array. This may result from deletions spanning a polymorphic site $[33,34]$, secondary polymorphisms interfering with genotyping at the primary polymorphic target (as was observed for two markers with Sanger sequencing of the C. maxima and C. medica accessions), and even unexpected alleles at the

Table 6 Mappable loci for comparative genetic mapping.

\begin{tabular}{|c|c|c|c|c|c|c|c|c|c|c|}
\hline & Clem & C. max. & C. med. & C. ret. & C. arf & C. aur. & C. $\lim$. & C. par. & C. $\sin$. & C. mic. \\
\hline Clementine & 567 & 36 & 13 & 67 & 51 & 13 & 58 & 6 & 26 & 2 \\
\hline C. maxima & 136 & 189 & 9 & 40 & 27 & 15 & 37 & 5 & 18 & 2 \\
\hline C. medica & 73 & 28 & 99 & 16 & 17 & 5 & 16 & 3 & 4 & 1 \\
\hline C. reticulata & 444 & 122 & 73 & 554 & 63 & 17 & 76 & 9 & 26 & 2 \\
\hline C. aurantifolia & 271 & 78 & 52 & 264 & 377 & 15 & 63 & 8 & 21 & 2 \\
\hline C. aurantium & 264 & 64 & 47 & 260 & 207 & 295 & 15 & 5 & 11 & 2 \\
\hline C. limon & 350 & 88 & 63 & 350 & 263 & 256 & 460 & 9 & 23 & 2 \\
\hline C. paradisis & 192 & 57 & 38 & 193 & 142 & 139 & 171 & 221 & 7 & 1 \\
\hline C. sinensis & 321 & 101 & 49 & 301 & 216 & 208 & 269 & 192 & 361 & 2 \\
\hline C. micrantha & 14 & 6 & 6 & 15 & 13 & 11 & 13 & 10 & 10 & 17 \\
\hline Total WONA & 476 & 141 & 79 & 461 & 295 & 277 & 375 & 211 & 332 & 15 \\
\hline Total WNA & 91 & 48 & 20 & 93 & 82 & 18 & 85 & 10 & 29 & 2 \\
\hline
\end{tabular}

Above the diagonal, loci with null alleles (WNA) polymorphic within each species; below the diagonal, loci without null alleles (WONA) polymorphic within each species; diagonal in bold, total number of loci mappable in one species (both WNA and WONA). 
primary polymorphism (such as triallelic sites; [35]). All of these are important potential sources of reproducible, but inaccurate, genotypes for population genetic studies because heterozygous null alleles are indistinguishable from the expected homozygotes on most genotyping platforms. In this study, among the high quality markers, 506 WONA and 116 WNA loci were selected. The transferability of SNPs to the whole Citrus genus can thus be estimated as 506/622 $=81.4 \%$ if all loci are considered, or as $480 / 596=80.5 \%$ considering loci identified in the Clementine BES. The null alleles were primarily found in C. medica, C. maxima, and wild Citrus with an average homozygosity frequency for null alleles being $0.64,0.61$ and 0.47 , respectively, but only 0.11 in C. reticulata. As these markers were identified from the Clementine BES and GoldenGate primers defined from Clementine sequences, these results are reasonable based on the strong genetic relationship between Clementine and $C$. reticulata and the important inter-specific differentiation between $C$. reticulata and the others basic taxa [28]. For the secondary species resulting from hybridization between the $C$. reticulata gene pool and the other basic taxa, the WNA loci present the advantage of frequent recessivity for the other parental gene pools. Therefore, it may allow identification of the $C$. reticulata subset that contributed to the secondary species genesis. Moreover, heterozygous null alleles should be useful for genetic mapping.

SNPs mined in a single heterozygous genotype provide a distorted view of the gene pool diversity but confirm the high stratification of the Citrus genus

The selection of heterozygous markers in Clementine primarily affects the estimation of two components of genetic diversity. The first is the differentiation between the basic taxa that appears to be underestimated with $\mathrm{CHet}$ loci when compared with $\mathrm{CHom}$ loci. The second is the intra-specific diversity. C. reticulata within diversity (both intercultivar and heterozygosity) and C. sinensis heterozygosity appear to be overestimated using the $\mathrm{CHet}$ loci when compared with the CHom loci. This is in contrast with the results obtained for C. paradisi, $C$. aurantifolia and C. limon. Previous studies have shown that Clementine is highly related to $C$. reticulata with a limited introgression of C. maxima $[24,28,36]$. Therefore, it can be inferred that the majority of the Clementine heterozygosity arose from the $C$. reticulata gene pool diversity. This may explain the overestimation of $C$. reticulata diversity when compared to C. maxima and C. medica, as well as the underestimation of the Citrus gene pool stratification. The genetic constitution of $C$. sinensis (mainly issued from the $C$. reticulata gene pool; see below for more detail) may also explain its higher heterozygosity for the CHet markers. According to their supposed origin (see below), the heterozygosity of $C$. limon and $C$. aurantifolia is based on the interspecific differentiation between the basic taxa. On the other hand, C. paradisi arose from C. maxima/C. reticulata differentiation and C. maxima within diversity. Thus, the underestimation of interspecific differentiation and the underestimation of C. maxima within diversity with the CHet markers explain the underestimation of diversity and heterozygosity of the above mentioned secondary species. In the present study, very low intraspecific polymorphism was identified for C. maxima and $C$. medica, regardless of the heterozygosity of the markers in Clementine. However, previous SSR studies reported similar within species diversity in C. maxima and $C$. reticulata $[27,28]$. It is highly probable that the set of markers used in this study target primarily within $C$. reticulata polymorphisms from one side and interspecific polymorphisms from the other side. Overall, the results reported here illustrate the limit and bias of the SNP array approach for large diversity analysis on a highly stratified population when the SNP discovery is based on a very limited panel. The SNP ascertainment bias has been widely discussed in humans [10,12] and animals [11,13-15] in relation with geographical stratification. This study reports bias associated with 'racial' differentiation in cultivated plants. This bias is enforced when taking into account the WNA loci. Future accurate analysis of the interspecific mosaic structure of secondary species and intra- and interspecific polymorphism analysis should be based on a non-biased pangenomic set of markers. The availability of a reference citrus genome sequence $[29,30]$ and the new sequencing methodologies will soon allow these objectives to be re-visited by the resequencing of several accessions of the basic taxa and secondary species.

Despite the distorted view of the gene pool diversity, the global organization around the basic taxa is still clear in both the PCA and NJ representation based in the WONA loci. The analysis of Fstat parameters on the subset of the genotypes of the three basic taxa ( $C$. reticulata, C. medica, C. maxima) with a non-significant Fis value but high Fit and Fst values confirms this important organization of the allelic diversity between these taxa. Moreover, a very high proportion of loci pairs display significant linkage disequilibrium in the germplasm sample. The majority of these locus pairs with significant LD in the germplasm sample appear in equilibrium within the segregating 'Chandler pummelo $\times$ Nules Clementine' $(\mathrm{CxN})$ population, testifying for very extended LD in the Citrus genus. Similar results were observed by Garcia-Lor et al. [28] for SSRs and InDel markers with significant LD for loci situated in different linkage groups. Breeding systems and domestication history are determinant factors of the LD structure in the 
germplasm of cultivated plants $[37,38]$. The extent of $\mathrm{LD}$ is generally greater for species that possess a selfing mating system [39-41] than for outcrossing ones [42-44]. The heterozygous deficit and generalized linkage disequilibrium observed in the Citrus genus indicates a strong population subdivision and thus a low gene flow between C. medica, C. reticulata, C. maxima and wild Citrus. The differentiation between these sexually compatible taxa may be explained by foundation effect in three geographic zones and by an initial allopatric evolution. C. maxima originated in the Malay Archipelago and Indonesia, C. medica evolved in northeastern India and the nearby region of Burma and China, and C. reticulata diversification occurred over a region including Vietnam, southern China and Japan $[20,45]$. Secondary species arose from the hybridization of the basic taxa. The partial apomixis of most of the secondary species has certainly been an essential element in the limitation of gene flows after that human activities have put into contact the differentiated gene pools of the basic taxa. Apomixis may also explain that, in agreement with previous molecular studies $[27,28,46]$, very few polymorphisms were found between the analyzed genotypes within $C$. sinensis, $C$. aurantium and C. paradisi although they were highly heterozygous (Ho of $0.65,0.55$ and 0.42 , respectively, with the whole set of WONA markers). This confirms that most of the intraspecific polymorphism of these secondary species arose from punctual mutation, transposable element movement [47] or epigenetic variation.

\section{Some parentage hypotheses for secondary species are strongly comfirmed}

The parentage hypothesis of some very important commercial species or cultivars was checked using their position in the PCA and NJA representations and the loci count for which the hybrid genotype disagrees with the supposed parent ones (data not shown based on the 506 WONA loci). The synthesis of the parentage hypothesis is given in Figure 6.

Sweet orange (C. sinensis) and sour orange (C. aurantium): these two species are believed to derive from hybridizations between the C. maxima and C. reticulata gene pools $[24,27,48]$. Their positions in the PCA analysis with C. reticulata and C. maxima used as active individuals confirm that a predominant portion of their

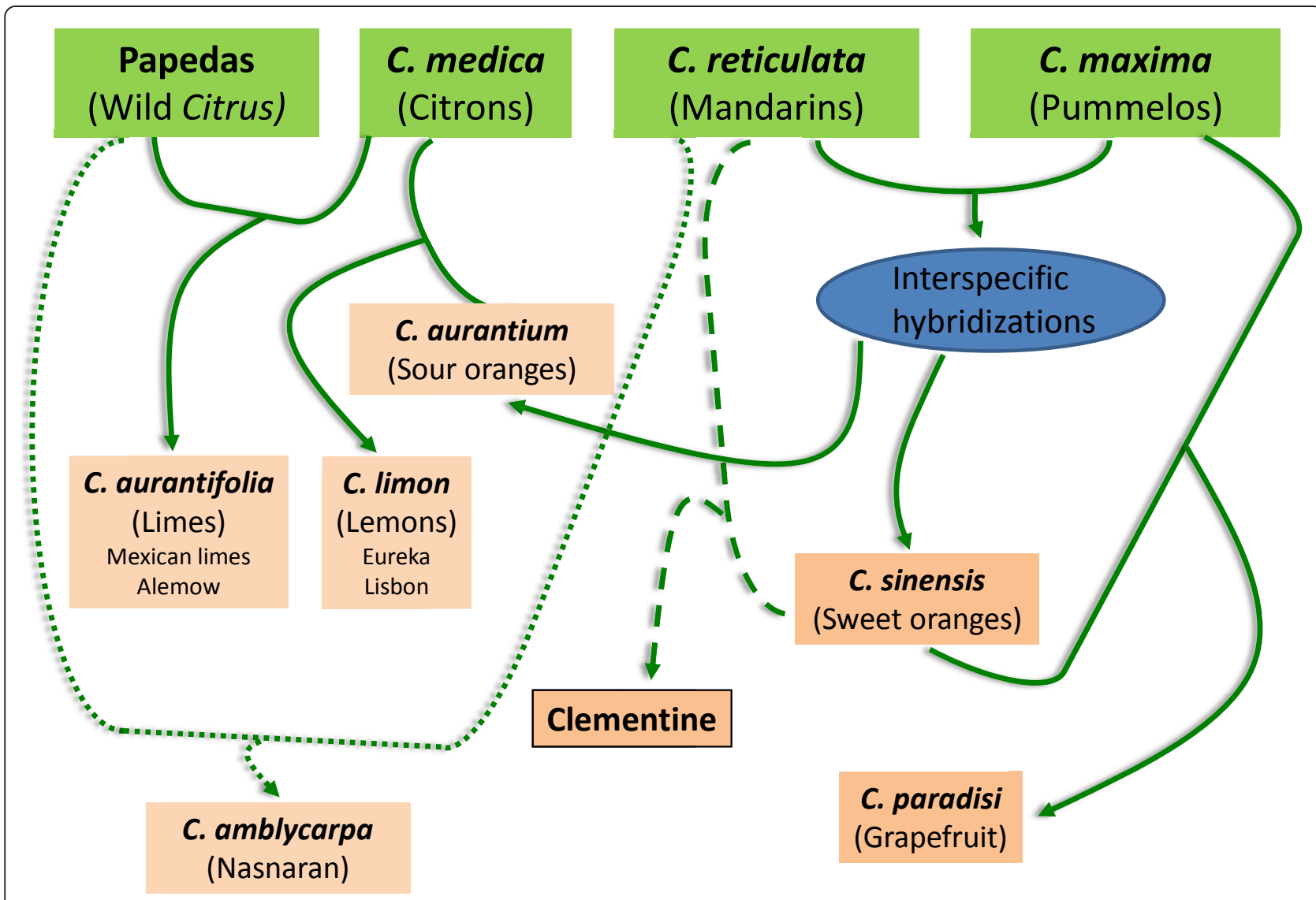

Figure 6 Hypothesis on the origin of some secondary Citrus species. 
genome arose from the $C$. reticulata gene pool, as previously deduced from SSR markers [28,49].

Clementine: it is generally agreed that Father Clement selected, a little more than one century ago in Algeria, Clementine as a chance seedling from 'Mediterranean' mandarin. The mandarin female parentage was confirmed by mitochondrial genome analysis [50]. 'Granito' sour orange was initially considered to be the male parent. However, previous molecular studies suggested that Clementine was more likely a mandarin $\times$ sweet orange hybrid $[24,36]$. The position of Clementine relative to sweet orange, sour orange and 'Mediterranean' mandarin in the PCA analysis supports this hypothesis. The hypothesis of a 'Mediterranean' mandarin $\times$ sweet orange is definitively authenticated with only one locus out of 506 suggesting incompatible genotypes, while 86 loci disagree with the hypothesis of an hybridization between 'Mediterranean' mandarin and sour orange.

Grapefruit (C. paradisi): the origin of grapefruit is attributed to a natural hybridization between pummelo (C. maxima) and sweet orange (C. sinensis). This hybridization may have occurred in the Caribbean more than 200 years ago [21,51-53]. In this study, grapefruit had an intermediary position between the sweet orange and pummelo gene pool in the PCA representation. Moreover, the NJA for the WNA markers clearly demonstrates the relationship of grapefruit and sweet orange. According to the sweet orange/pummelo combination, $96.3 \%$ to $98.0 \%$ of the 506 analyzed markers agree with this hypothesis. The best fitting is found with Tahiti pummelo. When searching for a potential sour orange $x$ pummelo origin, the percentage of loci in disagreement varies between $12.5 \%$ and $14.5 \%$. C. maxima is a polymorphic non-apomictic species. Therefore, due to the limited number of pummelo accessions analyzed, the absence of a $100 \%$ fit is reasonable. Moreover, as previously discussed, null alleles are relatively frequent in pummelo and it is likely that some of them have not been identified due to their heterozygous status. Therefore, the absence of some parental alleles in grapefruit, although they seem homozygous in one of the parents, may be explained by heterozygous null alleles for this parent. Upon looking for alleles present in grapefruit but absent in the two potential parents, only one to four loci disagree according to the considered pummelos. Thus, the data strongly confirm the hypothesis of the sweet orange $\times$ pummelo hybridization.

'Eureka' and 'Lisbon' lemon: Based on RFLP, RAPD and CAPS data, Nicolosi et al. [24] were the first to propose that lemon arose from a hybridization between $C$. aurantium and $C$. medica. This hypothesis was supported by nuclear SSR analysis [28]. In the present study, 'Eureka' and 'Lisbon' lemon varieties were highly heterozygous and very similar. These lemons are very likely two somatic mutants of the same ancestor. In PCA, their position was between the C. aurantium and C. medica group in each factorial axis. According to the citron accession, $96.0 \%$ to $97.8 \%$ of the 506 analyzed markers agree with this hypothesis. Moreover, null alleles are relatively frequent in citron and pummelo (contributing to sour-orange genesis). As for grapefruit, a search for alleles present in lemon but absent in the two potential parents reveals only one locus (CiC484104) out of 506 that disagrees with this hypothesis.

Mexican lime and Alemow: these two citrus were considered two distinct species, $C$. aurantifolia and $C$. macrophylla, respectively, by Tanaka [54]. However, Swingle and Reece [19] joined the two in C. aurantifolia. In all of the analysis reported here, these two were very close and intermediary between the citron cluster and a papeda cluster including $C$. hystrix and $C$. micrantha. For Mexican lime, this position is in agreement with the hypothesis proposed by Nicolosi et al. [24]. These authors proposed that Mexican lime was a hybrid between $C$. micrantha x $C$. medica. The maternal phylogeny was recently confirmed by Froelicher et al. [50]. According to the citron cultivar, $97.4 \%$ to $98.5 \%$ of the 506 analyzed markers agree with the C. micrantha $\mathrm{x}$ C. medica hypothesis with 'Humpang' citron providing the best fit. Moreover, no allele observed in Mexican lime was absent in the two potential parents. A $C$. hystrix x C. medica hypothesis produced very close results $(97.3 \%$ to $97.7 \%)$. Very similar results were observed for 'Alemow' with $96.7 \%$ to $97.4 \%$ and $96.0 \%$ and $96.9 \%$ of loci in agreements with $C$. micrantha $\mathrm{x} C$. medica and C. hystrix x C. medica origins, respectively. The papeda maternal parentage of Alemow was demonstrated by mitochondrial genome analysis [50]. Mexican lime and Alemow clearly have similar origins, and the papeda $\times C$. medica hypothesis was confirmed by this data. An enhanced study of papeda germplasm will be necessary to definitively conclude $C$. micrantha or another papeda as the female parent.

C. amblycarpa is native to Indonesia where it is called Djerook leemo (http://www.ars-grin.gov/cgi-bin/npgs/ html/taxon.pl?10679). It is generally considered to be a mandarin hybrid, and its common English name is Nasnaran mandarin. However, Froelicher et al. [50] showed that it has a papeda mitotype, identical to C. micrantha and C. hystrix. In PCA, C. amblycarpa displays an intermediary position between the two mentioned papedas and the acidic mandarin group ('Depressa', 'Sunki', 'Cleopatra' and 'Sun Chu Cha' mandarins). Its relationship with these mandarins is confirmed by the sharing of the same cluster in the NJA based on the WNA loci. A search for a potential direct papeda $\times$ C. reticulata origin was conducted. The best observed fit was a $C$. amblycarpa $\mathrm{x}$ 'Sun Chu Cha' mandarin hybridization 
with $92.5 \%$ of loci in agreement. For only $0.4 \%$ of the loci, one allele absent in the two parents was found in C. amblycarpa. Therefore, the hypothesis of papeda $x$ acidic mandarin is proposed for C. amblycarpa.

\section{A very useful set of SNPs markers for the comparative genetic mapping in Citrus and association genetic studies in C. reticulata}

Compared with other crops, genetic mapping in citrus is still undeveloped. The recent genetic maps based on codominant markers (primarily SSRs) [55-57] integrated around 150 markers, while maps based on dominant markers such as AFLPs [58], SRAPs, ISSRs, and RAPDs [59] included a little more than 200 markers. The markers mined in Clementine BES will be very useful for genetic mapping and association genetics in C. reticulata (554 polymorphic loci). The usefulness of these markers is more limited for the other basic taxa with 189, 99 and 17 polymorphic markers within C. maxima, C. medica and C. micrantha, respectively. For the secondary species, the marker number varies from 221 for C. paradisi to 460 for C. limon. For C. sinensis, C. aurantium and C. paradisi, where very little inter-cultivar diversity was found, the numbers of polymorphic loci are very similar to the number of heterozygous loci in a concrete genotype (and thus, directly mappable from a segregating population having such a genotype as a parent). For the other species, a consensus map should be established from several progenies to map all of the indicated markers. Moreover, it is probable that secondary species, such as $C$. paradisi, C. sinensis, C. aurantium and C. limon, have numerous heterozygous null alleles (inherited from the C. maxima or C. medica gene pools) for WNA loci in phylogenetic heterozygosity $(C$. reticulata/C. maxima or C. reticulata/C. medica). Therefore, the real number of mappable loci should be higher for these species. The biallelic nature of SNP markers limits the possibility to establish two anchored maps (male and female) from a single cross. This is because the allelelic phase of the markers heterozygous for the two parents can only be inferred for homozygous progenies. Multiallelic markers like SSRs are more powerful for such application [46].

The 547 markers heterozygous in Clementine are currently mapped in the framework of the International Citrus Genome Consortium (ICGC; [60]) and will contribute to the assembly of the reference citrus whole genome sequence. Interestingly, at least 346 of these markers should also be mapped on sweet orange in order to anchor the sweet orange genetic map developed by an US consortium [57] and the Clementine map to establish a saturated consensus citrus genetic map. Furthermore, it is also notable to mention that a large proportion of the analyzed SNPs are located in or close to putative coding regions [31]; therefore, these 'functional SNPs' may provide an important resource for the identification of genes associated with specific trait loci.

\section{Conclusions}

A set of 622 SNP markers providing consistent results was selected. Of the selected markers mined in Clementine BES, $80.5 \%$ were successfully transferred to the whole Citrus gene pool. The 116 loci with incomplete transferability displayed null allele homozygotes primarily in C. medica, C. maxima and wild Citrus species. The recessivity of the null alleles from these basic species should be useful in the identification of the subgene pools of $C$. reticulata at the origin of several interspecific hybrid species or varieties. Heterozygous null alleles should be useful for genetic mapping, particularly in secondary species. The two main biases associated with the SNP mining in Clementine were an overestimation of $C$. reticulata diversity and an underestimation of interspecific differentiation. However, the organization of the gene pool remained important, with high interspecific Fst values and very frequent significant linkage disequilibrium between markers pairs in equilibrium in the segregating population. Thus, despite the ascertainment biases, the SNP data confirms the important stratification of the gene pools around C. maxima, C. medica and $C$. reticulata, as well as the previous hypothesis on the origin of secondary species. The shared intra-specific polymorphism and accession heterozygosity will permit interspecific comparative genetic mapping. The implemented SNP marker set will also be very useful for association genetic studies in C. reticulata.

\section{Methods}

\section{Plant material}

In addition to Clementine cv 'Nules' (whose BES were used for SNP mining), 53 varieties from the citrus germplasm bank of IVIA (Spain) and INRA/CIRAD (France) were used for the transferability and diversity study of SNPs within the Citrus Genus. According to the Swingle and Reece classification [19] and the Nicolosi et al. [24] hypothesis on the origin of cultivated citrus species, 29 belong to the three primary species $(12 \mathrm{C}$. reticulata, 10 C. maxima and seven C. medica), 19 represent secondary species (two C. aurantium, four C. sinensis, two C. paradisi, seven C. limon and four C. aurantifolia), and five are wild species (additional file 6). Among the C. medica accessions, the present work confirmed the previously doubted classification of two cultivars as true citron ('Damas' and 'Rhob el Arsa' cultivars). These cultivars were thus excluded from the evaluation of within species diversity and between species organization of diversity.

A segregating population of 52 interspecific hybrids of 'Chandler' pummelo $\times$ 'Nules' Clementine $(\mathrm{CxN}$, 
developed by Cirad in Corsica) was used to confirm Clementine heterozygosity. This population was helpful in making the genotypic assignments of the germplasm samples and in comparing the linkage disequilibrium (LD) distribution of the germplasm (depending on the evolutionary history of the gene pool and marker linkage) and the segregating population (depending only on the marker linkage).

\section{DNA extraction}

Total DNA was extracted from fresh leaves according to Doyle and Doyle [61].

\section{SNP mining from Clementine BACend sequences}

As described in Terol et al. [31], in silico SNP mining was performed from 46,339 C. clementina cv. Nules BACend sequences (BESs) covering 28.1 Mb of genomic sequences. Assembly of BESs that did not contain repetitive sequences was performed using CAP3 [62]. A total of 6,461 contigs, including 19,057 reads and covering $6.14 \mathrm{Mb}$ of sequence, were produced. The SNPs were mined in these contigs using POLYBAYES software. A total of 6,617 putative SNPs (1.08 SNPs per kb) were found. A total of 4,904 SNPs were in silico tested for their potential use in the Illumina Golden Gate array following the Illumina procedure.

\section{SNP mining in candidate genes}

In an effort to identify SNPs within the Citrus genus, two cultivars of $C$. medica (Corsican and Budha's hand citrons), two cultivars of $C$. reticulata ('Cleopatra' and 'Mediterranean' mandarin), two cultivars of C. maxima ('Chandler' and 'Pink' pummelos) and one C. micrantha accession were selected. Primers (additional file 2) were defined from EST sequences available in the public databases for six genes implicated in primary and secondary metabolite biosynthesis pathways involved in determining citrus fruit quality (sugars, acids, flavonoids and carotenoids: Chalcone isomerase -CHI-, Vacuolar citrate/H + symporter -TRPA-, Phosphofructokinase -PKF-, Lycopene $\beta$-cyclase -LCY2-, Phytoene synthase -PSY-, Lycopene $\beta$-cyclase -LCYB-) and four candidates genes linked to salt tolerance (Cation/H+ antiporter -CAX-, Ascorbate oxydase -AOC-, High-affinity K+ Transporter 1 -HKT1- and Tréhalose-6-Phosphate Synthase -TS-). PCR amplifications of the samples were performed using a Mastercycler EP Gradient $S$ thermocycler (Eppendorf) in a $100 \mu \mathrm{L}$ final volume containing 0.025 $\mathrm{U} / \mu \mathrm{L}$ of Pfu DNA polymerase (Fermentas), $2 \mathrm{ng} / \mu \mathrm{L}$ of genomic DNA, $0.2 \mathrm{mM}$ of each dNTP, $2 \mathrm{mM} \mathrm{MgSO}_{4}$, $75 \mathrm{mM}$ Tris- $\mathrm{HCl}$ (pH 8.8), $20 \mathrm{mM}\left(\mathrm{NH}_{4}\right)_{2} \mathrm{SO}_{4}$, and 0.2 $\mu \mathrm{M}$ of each primer. The following PCR program was used: denaturation at $94^{\circ} \mathrm{C}$ for $5 \mathrm{~min}$; 35 repetitions of $30 \mathrm{~s}$ at $94^{\circ} \mathrm{C}, 1 \mathrm{~min}$ at $55^{\circ} \mathrm{C}$ or $60^{\circ} \mathrm{C}$ (according to primer $\mathrm{T}_{\mathrm{m}}$ ), and $45 \mathrm{~s}$ at $72^{\circ} \mathrm{C}$; and a final elongation step of $4 \mathrm{~min}$ at $72^{\circ} \mathrm{C}$. PCR product purification was performed using the QIAquick ${ }^{\circledR}$ PCR Purification Kit (Qiagen). Amplicons of the seven selected genotypes were sequenced by the Sanger method from the 5' extremity using dideoxynucleotides marked with fluorescence (Big Dye Terminator Cycle Sequencing Kit v3.1). Sequences were aligned with BioEdit [63] and SNPs were detected along the sequences. Of the identified polymorphisms, 121 were in silico tested for their potential use in the Illumina GoldenGate assay.

\section{SNP genotyping with an Illumina GoldenGate microarray}

For each selected SNP locus, three primers were designed using the Illumina Assay Design Tool (https:// icom.illumina.com). Sequence and primer information for the selected SNPs are listed in the additional file 1. The DNA of the samples to be genotyped was quantified according to Illumina specifications using PicoGreen (Molecular Probes) and a Gemini XPS Fluorescence Microplate Reader (Molecular Devices). The genotyping reactions were performed according to the standard Illumina GoldenGate assay instructions (http://www.illumina.com). In brief, $250 \mathrm{ng}$ of template DNA was used per sample. SNP-specific oligonucleotides containing both detection specific sequences and universal primer sequences were hybridized, extended and ligated to a common oligonucleotide containing a universal primer sequence. Ligated products were amplified using a universal primer set. Genotypes were determined by hybridizing the amplified products to a bead array which was complementary to the sequence specific tags. The fluorescence of the bead array was determined using a Bead Array Reader (Illumina). Two genotype controls ('Nules' Clementine and 'Chandler' pummelo) were repeated twice in each plate. The data were collected and analyzed using the Genome Studio software (Illumina). The automatic allele calling was visually checked and corrected if necessary, taking advantage of the segregating Pummelo $\times$ Clementine population.

\section{SNP genotyping validation by Sanger sequencing of amplicons}

Of the 54 Citrus accessions genotyped with the GoldenGate array, 24 were used to validate the genotyping data for 15 SNPs from five genes ( $L C Y 2, L C Y b, P K F, P S Y$, TRPA). This subset included 'Nules' Clementine, seven accessions of $C$. reticulata, five C. maxima, four C. med$i c a$, two C. aurantium, one C. sinensis, one C. paradisi, one C. limon, one C. aurantifolia, and one C. micrantha (additional file 6). The primers and PCR amplification, purification and sequencing were the same as that used for the SNP mining in the candidate genes. 


\section{Study of the origin of unexpected SNPs by Sanger analysis}

The origin of unexpected polymorphisms displayed by several SNP markers from the Clementine BES, such as null alleles, no heterozygosity for Clementine and 'fixed heterozygosity', was analyzed by Sanger sequencing of the amplicons of four accessions: 'Nules' Clementine, haploid Clementine, 'Chandler' pummelo and Corsican citron. Primers flanking the SNP site were defined from the contig sequences obtained from the BES [31] to produce amplified fragments ranging from 200 to $620 \mathrm{bp}$ (additional file 7). PCR amplification, purification and sequencing were performed in the same manner as the SNP mining in the candidate genes.

\section{Data analysis}

Neighbor-joining analysis [64] was computed using DARwin software version 5.0 [65]. Genetic dissimilarities were calculated using the simple matching dissimilarity index $\left(\mathrm{d}_{\mathrm{i}-\mathrm{j}}\right)$ between pairs of accessions:

$$
d_{i-j}=1-1 / L \sum_{l=1}^{L} m_{l} / 2
$$

with $d_{i-j}$, the dissimilarity between units i and $j$; $L$, the number of loci; $\mathrm{m}_{\mathrm{l}}$, the number of matching alleles for locus 1 . Weighted neighbor-joining trees were computed from the dissimilarity matrix with 1000 bootstraps to test branch robustness. Principal Component Analyses (PCA) were computed using XLSTAT on the matrix of the frequencies for each allele. Genetic population parameters (Ho, observed heterozygosity; He, expected heterozygosity equivalent to Nei diversity index [66]; and Fstats - Fis, Fit and Fst - based on the parameters of Wright [67] and Weir \& Cockerham [68] were calculated with GENETIX v. 4.03 software.

For each locus with null alleles, the genotypic diversity (GD) was estimated as

$$
G D=1-\sum_{l=1}^{G} g_{i}^{2}
$$

with $\mathrm{G}$ indicating the total number of observed genotypes and $g_{\mathrm{i}}$ indicating the frequency of each observed genotype.

Linkage disequilibrium (LD) was estimated by $\mathrm{r}^{2}$ (chi square Pearson's correlation coefficient). The significances were estimated with the exac-test pvalue using PowerMarker software v. 3.25 [69].

\section{Additional material}

Additional file 1: Information on SNP markers included in the GoldenGate array. This file contains the main information on the SNP markers included in the GoldenGate array. It includes GenBank accession number, sequence surrounding the SNPS, SNP position, GoldenGate primers and designability rank, genotyping code, identification of the loci used for the diversity study.

Additional file 2: Primers for SNP mining in candidate genes and polymorphism results for 7 genotypes representative of 4 basic Citrus taxa. This file contains the main information on the primers used for SNP mining in candidate gene sequences (GenBank accession number, primer sequences, annealing temperature and theoretical amplicon size from EST data) and result data (size of exploitable sequence, number and frequency of SNPS).

Additional file 3: Detailed diversity results for loci without null allele (WONA). This file contains main data on the results obtained with WONA loci. It includes heterozygosity in Clementine, observed and theoretical heterozygosity in the whole population and each species, Fstat parameters in the whole population and between and within the three main species (C. reticulata, C. medica, C. maxima) and structuration level between $C$. reticulata and C. maxima.

Additional file 4: Additional figures. This file contains two figures. Figure S1: Correlation between the contribution of SNPs loci to the first axis of PCA analysis (C. maxima and C. reticulata as active individuals) and the Fst values for C. maxima/C. reticulata differentiation. Figure S2: Co-distribution of LD between loci without null allele (WONA) pairs for Chandler $\times$ Clementine progeny and germplasm population

Additional file 5: Detailed diversity results for loci with null allele (WNA). This file contains main data on the results obtained with WNA loci. It includes (i) SNP heterozygosity and heterozygosity for null allele in Clementine, (ii) number of individual in heterozygosity (SNPs),

homozygous for one SNP allele and homozygous for null allele in the whole population and within the different species, (iii) genotypic diversity in the whole population (PIC).

Additional file 6: List of germplasm analyzed. This file contains the list of citrus germplasm accession analyzed. It includes the gerplasm bank, the accession number, the varietal group, the common name, the Latin name according Swingle and Reece and Tanaka classifications and the use for SNP genotyping validation by sequencing.

Additional file 7: Primers for analyzing the origin of unexpected genotyping in the GoldenGate array. this file contains the main information on the primers used for analyzing the origin of unexpected genotyping in the GoldenGate array. It includes the locus name, the abnormality type, the primer sequences, annealing temperature and amplicon size.

\section{Acknowledgements}

This work was founded by the French Genomic ANR 2008 CITRUSSEQ project

\section{Author details}

'CIRAD, UMR AGAP, Avenue Agropolis, TA A-108/02, 34398 Montpellier, Cedex 5, France. ${ }^{2}$ Centro de Proteccion Vegetal y Biotechnologia, IVIA, Apartado Oficial,46113 Moncada (Valencia), Spain. ${ }^{3}$ Centro de genomica, IVIA, Apartado Oficial, 46113 Moncada (Valencia), Spain. ${ }^{4}$ INRA, UR EPGV, 2 rue Gaston Cremieux, 91057, Evry, France.

\section{Authors' contributions}

PO has managed the work, analyzed the data and wrote the paper. JT and MT have generated the Clementine BES data, selected the SNPs from the Clementine BES and contributed to the data validation. $A G L, C L$ and $R M$ have identified SNPs in the candidate genes. DB, AB and AC have developed the GoldenGate array and the SNP genotyping. YF has developed the Chandler $\times$ Clementine population and provided DNA. LN has provided most of the germplasm and contributed to the writing of the paper. All authors read and approved the final manuscript. 


\section{References}

1. Brookes AJ: The essence of SNPs. Gene 1999, 234(2):177-186.

2. Edwards D, Batley J: Plant genome sequencing: applications for crop improvement. Plant Biotechnology Journal 2010, 8(1):2-9.

3. Chen D, Ahlford A, Schnorrer F, Kalchhauser I, Fellner M, Viragh E, Kiss I, Syvanen AC, Dickson BJ: High-resolution, high-throughput SNP mapping in Drosophila melanogaster. Nat Methods 2008, 5(4):323-329.

4. van Os H, Andrzejewski S, Bakker E, Barrena I, Bryan GJ, Caromel B, Ghareeb $B$, Isidore $E$, de Jong $W$, van Koert $P$, Lefebvre $V$, Milbourne $D$, Ritter $\mathrm{E}$, van der Voort JN, Rousselle-Bourgeois F, van Vliet J, Waugh $\mathrm{R}$, Visser RG, Bakker J, van Eck HJ: Construction of a 10,000-marker ultradense genetic recombination map of potato: providing a framework for accelerated gene isolation and a genomewide physical map. Genetics 2006, 173(2):1075-1087.

5. Troggio M, Malacarne G, Coppola G, Segala C, Cartwright DA, Pindo M, Stefanini M, Mank R, Moroldo M, Morgante M, Grando MS, Velasco R: A dense single-nucleotide polymorphism-based genetic linkage map of grapevine (Vitis vinifera L.) anchoring Pinot Noir bacterial artificial chromosome contigs. Genetics 2007, 176(4):2637-2650

6. de Bakker PI, Burtt NP, Graham RR, Guiducci C, Yelensky R, Drake JA Bersaglieri T, Penney KL, Butler J, Young S, Onofrio RC, Lyon HN, Stram DO, Haiman CA, Freedman ML, Zhu X, Cooper R, Groop L, Kolonel LN, Henderson BE, Daly MJ, Hirschhorn JN, Altshuler D: Transferability of tag SNPs in genetic association studies in multiple populations. Nat Genet 2006, 38(11):1298-1303.

7. McCouch SR, Zhao K, Wright M, Tung C, Ebana K, Thomson M, Reynolds A Wang D, DeClerck G, Ali ML, McClung A, Eizenga G, Bustamante C: Development of genome-wide SNP assays for rice. Breed Sci 2010, 60(5):524-535.

8. Nikiforov $\Pi$, Rendle RB, Goelet P, Rogers YH, Kotewicz ML, Anderson S, Trainor GL, Knapp MR: Genetic Bit Analysis: a solid phase method for typing single nucleotide polymorphisms. Nucleic Acids Res 1994, 22(20):4167-4175.

9. Sapolsky RJ, Hsie L, Berno A, Ghandour G, Mittmann M, Fan JB: Highthroughput polymorphism screening and genotyping with high-density oligonucleotide arrays. Genet Anal 1999, 14(5-6):187-192.

10. Clark AG, Hubisz MJ, Bustamante CD, Williamson SH, Nielsen R: Ascertainment bias in studies of human genome-wide polymorphism. Genome Res 2005, 15(11):1496-1502.

11. Rosenblum EB, Novembre J: Ascertainment bias in spatially structured populations: a case study in the eastern fence lizard. J Hered 2007, 98(4):331-336

12. Albrechtsen A, Nielsen FC, Nielsen R: Ascertainment biases in SNP chips affect measures of population divergence. Mol Biol Evol 2010, 27(11):2534-2547.

13. Negrini R, Mazza R, Colli L, Pellecchia M, Bomba L, Stella A, Williams $J$, Ajmone-Marsan P: Effect of non-random sampling of SNPs on the estimation of population genetic parameters. Proceedings of the 9th World Congress on Genetics Applied to Livestock Production (WCGALP): August 1-6, 2010; Leipzig. Germany 2010.

14. Trask JA, Malhi RS, Kanthaswamy S, Johnson J, Garnica WT, Malladi VS, Smith DG: The effect of SNP discovery method and sample size on estimation of population genetic data for Chinese and Indian rhesus macaques (Macaca mulatta). Primates 2011, 52(2):129-138.

15. Bradbury IR, Hubert $S$, Higgins B, Bowman S, Paterson IG, Snelgrove PV, Morris CJ, Gregory RS, Hardie DC, Borza T, Bentzen P: Evaluating SNP ascertainment bias and its impact on population assignment in Atlantic cod, Gadus morhua. Mol Ecol Resour 2011, 11(Suppl 1):218-225.

16. Vezzulli S, Micheletti D, Riaz S, Pindo M, Viola R, This P, Walker MA, Troggio M, Velasco R: A SNP transferability survey within the genus Vitis. BMC Plant Biol 2008, 8:128.

17. Carlson CS, Smith JD, Stanaway IB, Rieder MJ, Nickerson DA: Direct detection of null alleles in SNP genotyping data. Hum Mol Genet 2006 , 15(12):1931-1937.

18. Garvin MR, Saitoh K, Gharrett AJ: Application of single nucleotide polymorphisms to non-model species: a technical review. Mol Ecol Resour 2010, 10(6):915-934

19. Swingle WT, Reece PC: The botany of Citrus and its wild relatives. In The Citrus industry. Volume 1. University of California Press edition. Edited by: Reuther W, Webber HJ, Batchelor DL. Berkeley, CA, USA; 1967:190-430.
20. Scora RW: On the history and origin of Citrus. Bull Torr Bot Club 1975, 102:369-369-375.

21. Barrett HCRA: A numerical taxonomic study of affinity relationships in cultivated Citrus and its close relatives. Syst Bot 1976, 1:105-136.

22. Herrero R, Asins MJ, Pina JA, Carbonell EA, Navarro L: Genetic diversity in the orange subfamily Aurantioideae. II. Genetic relationships among genera and species. Theor App/ Genet 1996, 93(8):1327-1334.

23. Federici $C T$, Fang DQ, Scora RW, Roose ML: Phylogenetic relationships within the genus Citrus (Rutaceae) and related genera as revealed by RFLP and RAPD analysis. Theor App/ Genet 1998, 96(6/7):812-822.

24. Nicolosi E, Deng ZN, Gentile A, Malfa SI, Continella G, Tribulato E: Citrus phylogeny and genetic origin of important species as investigated by molecular markers. Theor App/ Genet 2000, 100(8):1155-1166.

25. Liang G, Xiong G, Guo Q, He Q, Li X: AFLP analysis and the taxonomy of Citrus. Acta Hort 760:137-142

26. Luro F, Rist D, Ollitrault P: Evaluation of genetic relationships in Citrus genus by means of sequence tagged microsatellites. Acta Horticulturae 2001, , 546: 237-242.

27. Barkley NA, Roose ML, Krueger RR, Federici CT: Assessing genetic diversity and population structure in a citrus germplasm collection utilizing simple sequence repeat markers (SSRs). Theor Appl Genet 2006, 112(8):1519-1531.

28. Garcia-Lor A: Comparative contribution of InDels and SSRs markers to decipher the interspecific structure of the cultivated Citrus genetic diversity; perspective for association genetic studies. MGG 2011, DOI: 10.1007/s00438-011-0658-4

29. Aleza P, Juarez J, Hernandez M, Pina JA, Ollitrault P, Navarro L: Recovery and characterization of a Citrus clementina Hort. ex Tan. 'Clemenules' haploid plant selected to establish the reference whole citrus genome sequence. BMC Plant Biol 2009, 9:110

30. Gmitter FG Jr: The haploid mandarin and diploid sweet orange genome sequences. Plant \& Animal Genomes XIX Conference, 15-19, 2011; San Diego, CA 2011.

31. Terol J, Naranjo MA, Ollitrault P, Talon M: Development of genomic resources for Citrus clementina: characterization of three deep-coverage BAC libraries and analysis of 46,000 BAC end sequences. BMC Genomics 2008, 9:423

32. Ollitrault $\mathrm{P}$, Jacquemond C, Dubois C, Luro F: Citrus. In Genetic diversity of cultivated tropical plants.. CIRAD edition. Edited by: Hamon P, Seguin M, Perrier X, Glaszmann JC. Montpellier; 2003:193-217.

33. Conrad DF, Andrews TD, Carter NP, Hurles ME, Pritchard JK: A highresolution survey of deletion polymorphism in the human genome. Nat Genet 2006, 38(1):75-81.

34. McCarroll SA, Hadnott TN, Perry GH, Sabeti PC, Zody MC, Barrett JC, Dallaire S, Gabriel SB, Lee C, Daly MJ, Altshuler DM, International HapMap Consortium: Common deletion polymorphisms in the human genome. Nat Genet 2006, 38(1):86-92.

35. Carlson CS, Eberle MA, Rieder MJ, Yi Q, Kruglyak L, Nickerson DA: Selecting a maximally informative set of single-nucleotide polymorphisms for association analyses using linkage disequilibrium. Am J Hum Genet 2004, 74(1):106-120

36. Deng ZN, Gentile A, Nicolosi E, Continella G, Tribulato E: Parentage determination of some citrus hybrids by molecular markers. Proc Int Soc Citricul 1996, 2:849-854.

37. Flint-Garcia SA, Thornsberry JM, Buckler ES: Structure of linkage disequilibrium in plants. Annu Rev Plant Biol 2003, 54:357-374

38. Abdurakhmonov IY, Abdukarimov A: Application of association mapping to understanding the genetic diversity of plant germplasm resources. Int J Plant Genomics 2008, 2008:574927.

39. Nordborg M, Borevitz JO, Bergelson J, Berry CC, Chory J, Hagenblad J, Kreitman M, Maloof JN, Noyes T, Oefner PJ, Stahl EA, Weigel D: The extent of linkage disequilibrium in Arabidopsis thaliana. Nat Genet 2002, 30(2):190-193.

40. Garris AJ, McCouch SR, Kresovich S: Population structure and its effect on haplotype diversity and linkage disequilibrium surrounding the xa5 locus of rice (Oryza sativa L.). Genetics 2003, 165(2):759-769.

41. Deu M, Glaszmann JC: Linkage disequilibrium in sorghum. Plant \& Animal Genomes XII Conference 10-14 January 2004; San Diego, CA 2004

42. Remington DL, Thornsberry JM, Matsuoka Y, Wilson LM, Whitt SR, Doebley J, Kresovich S, Goodman MM, Buckler ES: Structure of linkage 
disequilibrium and phenotypic associations in the maize genome. Proc Natl Acad Sci USA 2001, 98(20):11479-11484.

43. Ingvarsson PK: Nucleotide polymorphism and linkage disequilibrium within and among natural populations of European aspen (Populus tremula L., Salicaceae). Genetics 2005, 169(2):945-953.

44. Rafalski A, Morgante M: Corn and humans: recombination and linkage disequilibrium in two genomes of similar size. Trends Genet 2004, 20(2):103-111.

45. Webber HJ: History and development of the citrus industry. In The citrus industry. Volume 1. Edited by: Reuther W, Webber HJ. Berkeley: University of California Press; 1967:1-1-39.

46. Luro FL, Costantino G, Terol J, Argout X, Allario T, Wincker P, Talon M, Ollitrault P, Morillon R: Transferability of the EST-SSRs developed on Nules clementine (Citrus clementina Hort ex Tan) to other Citrus species and their effectiveness for genetic mapping. BMC Genomics 2008, 9:287.

47. Breto MP, Ruiz C, Pina JA, Asins MJ: The diversification of Citrus clementina Hort. ex Tan., a vegetatively propagated crop species. Mol Phylogenet Evol 2001, 21(2):285-293.

48. Uzun A, Yesiloglu T, Aka-Kacar Y, Tuzcu O, Gulsen O: Genetic diversity and relationships within Citrus and related genera based on sequence related amplified polymorphism markers (SRAPs). Scientia Horticulturae 2009, 121(3):306-312.

49. Roose ML, Federici CT, Mu L, Kwok K, Vu C: Map-based ancestry of sweet orange and other citrus variety groups. Second International Citrus Biotechnology Symposium 2009, 28.

50. Froelicher Y, Mouhaya W, Bassene JB, Costantino G, Kamiri M, Luro F, Morillon R, Ollitrault P: New universal mitochondrial PCR markers reveal new information on maternal citrus phylogeny. Tree Genetics and Genomes 2011, 7(1):49-61.

51. Webber HJ: Cultivated varieties of citrus. The Citrus Industry. History, World Distribution, Botany andVarieties. Volume 1 Anonymous California; 1943, 475-668, University of California Press edition.

52. de Moraes, Ana, Santos Soares Filho dos, Walter, Guerra, Marcelo: Karyotype diversity and the origin of grapefruit. Chromosome Research 2007, , 1: 115-121.

53. Scora RW, Kumamoto J, Soost RK, Nauer EM: Contribution to the origin of the grapefruit Citrus paradisi (Rutaceae). Syst Bot 1982, 7:170-177.

54. Tanaka T: Citologia: Semi-centennial Commemoration Papers on Citrus Studies Osaka, Japan: Citologia Supporting Foundation; 1961.

55. Bernet GP, FernandezRibacoba J, Carbonell EA, Asins MJ: Comparative genome-wide segregation analysis and map construction using a reciprocal cross design to facilitate citrus germplasm utilization. Mol Breed 2010, 25(4):659-673.

56. Chen C, Bowman K, Choi Y, Dang P, Rao M, Huang S, Soneji J, McCollum T, Gmitter F: EST-SSR genetic maps for Citrus sinensis and Poncirus trifoliata. Tree Genetics \& Genomes 2008, 4(1):1-10.

57. Lyon MT, Federici CT, Kacar Y, Chen C, O'Malley D, Chaparro JX, Gmitter FG, Roose ML: SSR-based linkage maps for sweet orange and trifoliate orange. Plant \& Animal Genome XVth Conference, January 13-17, 2007; San Diego, CA 2007.

58. Carlos de Oliveira A, Bastianel M, Cristofani-Yaly M, Morais do Amaral A, Machado MA: Development of genetic maps of the citrus varieties 'Murcott' tangor and 'Pera' sweet orange by using fluorescent AFLP markers. J Appl Genet 2007, 48(3):219-231.

59. Gulsen O, Uzun A, Canan I, Seday U, Canihos E: A new citrus linkage map based on SRAP, SSR, ISSR, POGP, RGA and RAPD markers. Euphytica 2010 173(2):265-277.

60. Ollitrault P, Terol J, Chen C, Federici CT, Lofty S, Hippolyte I, Ollitrault F, Berard A, Chauveau A, Constantino G, Kacar Y, Mu L, Cuenca J, Garcia-Lor A, Froelicher Y, Aleza P, Boland A, Billot C, Navarro L, Luro F, Roose ML, Gmitter FG, Talon M, Brunel D: A reference linkage map of C. clementina based on SNPs, SSRs and indels. Plant \& Animal Genomes XIX Conference, January 15-19, 2011; San Siego, CA 2011

61. Doyle JJ, Doyle JL: A rapid DNA isolation procedure for small quantities of fresh leaf tissue. Phytochemical Bulletin 1987, 19(1):11-11-15.

62. Huang X, Madan A: CAP3: A DNA sequence assembly program. Genome Res 1999, 9(9):868-877.

63. Hall TA: A user-friendly biological sequence alignment editor and analysis program for Windows 95/98/NT. Nucleic Acids Symp Ser 199 41:95-95-98.
64. Saitou N, Nei M: The neighbor-joining method: a new method for reconstructing phylogenetic trees. Mol Biol Evol 1987, 4(4):406-425.

65. DARwin software. [http://darwin.cirad.fr/darwin].

66. Nei M: Analysis of gene diversity in subdivided populations. Proc Natl Acad Sci USA 1973, 70(12 Pt 1-2):3321-3321-3323.

67. Wright S: Evolution and the genetics of populations. The theory of gene frequencies. Chicago: University of Chicago Press; 1969.

68. Weir BS, Cockerham CC: Estimating F-statistics for the analysis of population structure. Evolution 1984, 38(6):1358-1370.

69. Liu K, Muse SV: PowerMarker: an integrated analysis environment for genetic marker analysis. Bioinformatics 2005, 21(9):2128-2129.

doi:10.1186/1471-2164-13-13

Cite this article as: Ollitrault et al: SNP mining in C. clementina BAC end sequences; transferability in the Citrus genus (Rutaceae), phylogenetic inferences and perspectives for genetic mapping. BMC Genomics 2012 13:13.

\section{Submit your next manuscript to BioMed Central and take full advantage of:}

- Convenient online submission

- Thorough peer review

- No space constraints or color figure charges

- Immediate publication on acceptance

- Inclusion in PubMed, CAS, Scopus and Google Scholar

- Research which is freely available for redistribution

Submit your manuscript at www.biomedcentral.com/submit
Biomed Central 